University of Rhode Island

DigitalCommons@URI

Open Access Master's Theses

2014

\title{
COLLISION FREE PATH PLANNING OF AN OBJECT USING MULTIPLE ROBOT MANIPULATORS
}

Drew P. Davis

University of Rhode Island, drew_davis@my.uri.edu

Follow this and additional works at: https://digitalcommons.uri.edu/theses

\section{Recommended Citation}

Davis, Drew P., "COLLISION FREE PATH PLANNING OF AN OBJECT USING MULTIPLE ROBOT

MANIPULATORS" (2014). Open Access Master's Theses. Paper 373.

https://digitalcommons.uri.edu/theses/373

This Thesis is brought to you for free and open access by DigitalCommons@URI. It has been accepted for inclusion in Open Access Master's Theses by an authorized administrator of DigitalCommons@URI. For more information, please contact digitalcommons-group@uri.edu. 
COLLISION FREE PATH PLANNING OF AN OBJECT USING MULTIPLE ROBOT MANIPULATORS

BY

DREW P. DAVIS

A THESIS SUBMITTED IN PARTIAL FULFILLMENT OF THE

REQUIREMENTS FOR THE DEGREE OF

MASTER OF SCIENCE

IN

MECHANICAL ENGINEERING AND APPLIED MECHANICS

UNIVERSITY OF RHODE ISLAND

2014 
MASTER OF SCIENCE THESIS

$\mathrm{OF}$

DREW P. DAVIS

\title{
APPROVED:
}

Thesis Committee:

\author{
Major Professor Musa Jouaneh \\ David Chelidze \\ Richard Vaccaro \\ Nasser H. Zawia \\ DEAN OF THE GRADUATE SCHOOL
}

\section{UNIVERSITY OF RHODE ISLAND}

2014 


\begin{abstract}
This thesis presents two algorithms for the path planning of multiple manipulator systems attempting to transform a single object without the need for a closed form inverse kinematic solution for a specific manipulator. The first algorithm is a dual quaternion Jacobian based numerical inverse kinematic method. The Jacobian is formulated to transform the change in joint space of the manipulator to the change in dual quaternion transformation space of the manipulator's end effector. Joint space solutions are found that satisfy both the position and orientation of the end effector. The second algorithm is an adaptation of the sampling-based rapidly-exploring dense tree algorithm. The algorithm is adjusted to handle multiple manipulators cooperatively transforming a single object while avoiding environment collisions and invalid joint spaces. The tree generation algorithm forms extensions for the tree by extending the manipulators toward dual quaternion transformations of the object in the environment using the aforementioned numerical inverse kinematics algorithm. This avoids having to find random joint configurations of each manipulator that satisfy the closed link constraints caused by the grasps of each manipulator on the object. The algorithm is able to develop a path from an initial transformation to a goal transformation to the object using multiple robot manipulators. The algorithms presented are tested under a pair of simulation environments while varying the algorithm parameters as well as environment components such as the location of the manipulators as well as the presence of obstacles. Two seven degree of freedom Schunk manipulators were used for the experiments of this thesis.
\end{abstract}




\section{ACKNOWLEDGMENTS}

I would like to express my deepest thanks to my thesis advisor Dr. Musa Jouaneh for his guidance, patience, and support during the development of this thesis. His knowledge of robotic systems has been invaluable throughout the course of this work and has been a significant influence on my education.

I would also like to thank the other members of my thesis committee, Dr. David Chelidze from the Department of Mechanical, Industrial, and Systems Engineering, Dr. Richard Vaccaro from the Department of Electrical Engineering as well as the Mechanical Engineering department chair Dr. David Taggart for taking time out of their schedules to support my thesis defense.

I also would like to thank my loving family for their continuing support of all the endeavors I undertake in my private and professional life. 


\section{TABLE OF CONTENTS}

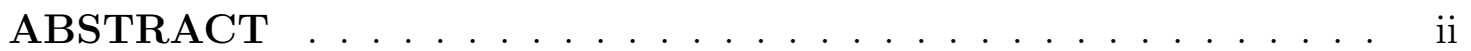

ACKNOWLEDGMENTS ................. . iii

TABLE OF CONTENTS ................... . iv

LIST OF TABLES . . . . . . . . . . . . . . . . . . vi

LIST OF FIGURES . . . . . . . . . . . . . . . . . . vii

\section{CHAPTER}

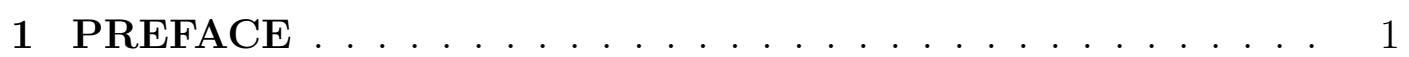

1.1 Introduction . . . . . . . . . . . . . . . . . 1

1.2 Review of Literature . . . . . . . . . . . . . . . . . . 4

1.3 Overview of Thesis $\ldots \ldots \ldots \ldots \ldots \ldots$

List of References . . . . . . . . . . . . . . . . . . . . 7

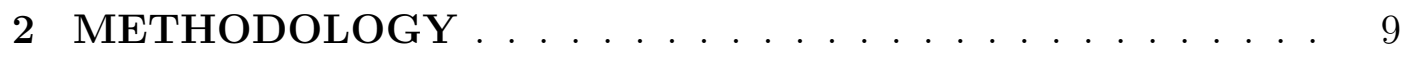

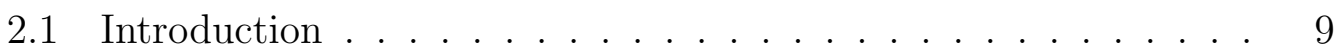

2.2 Transformation Representation . . . . . . . . . . . . . . . 10

2.3 Inverse Kinematics . . . . . . . . . . . . . . . . . . . . . 17

2.4 Cooperative Robot Manipulator Path Planning for the Transformation of an Object . . . . . . . . . . . . . . 19

List of References . . . . . . . . . . . . . . . . . . . . 28

3 FINDINGS $\ldots \ldots \ldots \ldots \ldots \ldots \ldots$

3.1 Simulation 1 Results . . . . . . . . . . . . . . . . . 31

3.2 Simulation 2 Results . . . . . . . . . . . . . . . . . . . . 38 


\section{Page}

3.3 Remarks . . . . . . . . . . . . . . . . . . . 45

List of References . . . . . . . . . . . . . . . . . 47

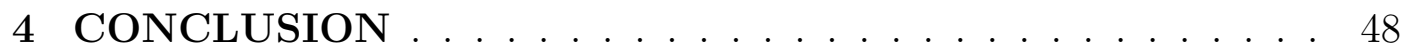

\section{APPENDIX}

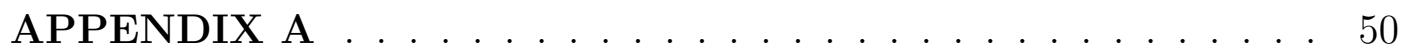

A.1 Forward Kinematics of the LWA3 Robot Manipulator . . . . . . 50

A.2 Jacobian Formulation of the LWA3 Robot Manipulator . . . . 53

List of References . . . . . . . . . . . . . . . . . . . 55

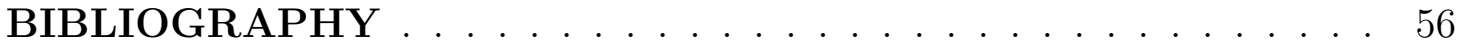




\section{LIST OF TABLES}

Table

1 Simulation 1 Tree and Path Generation Results with Modified

Parameters .................. . . 32

$2 \quad$ Simulation 1 Tree and Path Generation Results with Modified

Location of Manipulator 2 and Removed Obstacles . . . . . . . 33

3 Simulation 2 Tree and Path Generation Results with Modified

Parameters ................... . . 39

4 Simulation 2 Tree and Path Generation Results with Modified

Location of Manipulator 2 and Removed Obstacles . . . . . . . 40

A.1 Schunk LWA3 Joint Limits . . . . . . . . . . . . . . . . 51 


\section{LIST OF FIGURES}

$\begin{array}{lll}\text { Figure } & \text { Page }\end{array}$

$1 \quad$ Numerical Inverse Kinematics Algorithm . . . . . . . . . . . 19

2 Two Dimensional Configurations Space Rapidly-Exploring Dense Tree . . . . . . . . . . . . . . . . 20

3 Visualization of Vertices and Edges for Tree Generation . . . . . 20

4 Rapidly-Exploring Dense Tree Flowchart . . . . . . . . . . 23

$5 \quad$ Tree Generation Algorithm _. . . . . . . . . 27

$6 \quad$ Graphics Rendering Global Axes . . . . . . . . . . . . 29

$7 \quad$ Simulations 1 and 2 Initial and Sample Goal Configurations .. . 31

8 Simulation 1 Case 1 System Path with the Least Configurations 35

$9 \quad$ Simulation 1 Case 1 Joint Paths with the Least Path Configurations . . . . . . . . . . . . . . . 36

10 Simulation 1 System Path with the Most Configurations . . . . 37

11 Simulation 1 Case 1 Joint Paths with the Most Path Configurations . . . . . . . . . . . . . . . . 38

12 Simulation 2 System Path with the Least Configurations . . . . 42

13 Simulation 2 Case 1 Joint Paths with the Least Path Configurations . . . . . . . . . . . . . . . . . . 43

14 Simulation 2 System Path with the Most Configurations . . . . 44

15 Simulation 2 Case 1 Joint Paths with the Most Path Configurations . . . . . . . . . . . . . . . 45

A.1 Dimensions of the Schunk LWA3 Manipulator for Forward Kinematics . . . . . . . . . . . . . 50 


\section{CHAPTER 1}

\section{PREFACE}

\subsection{Introduction}

In the past few decades, robotic path planning has been a heavily researched topic. Due to the expensive nature of path planning with robot manipulators that have high degrees of freedom, heuristic planners or sampling-based algorithms have been developed. Research has been performed to extend planning of single manipulators to algorithms that plan multiple manipulators concurrently. Rapidlyexploring dense tree algorithms [1] have been successfully implemented for the collision free path planning of a manipulator from an initial configuration to a goal configuration in the presence of obstacles in a static environment. This study aims to extend the application of rapidly-exploring dense tree algorithms to an environment with multiple manipulators cooperatively manipulating a single rigid body object and ultimately return a path for the manipulators to follow that will transform the object from the initial state to a goal state.

Cooperative manipulation of an object offers multiple advantages over the use of a single manipulator for object manipulation. First and foremost, multiple robot manipulators are capable of distributing the load the object puts on a manipulator across several manipulators. Reduced loads on the joints of the manipulators allow smaller, cheaper manipulators to be used. Multiple manipulators also facilitate the transformation of bulky, obscure or awkward objects. Consider the manipulation of a table. With a single manipulator with a single grasp along the edge of the table, the transformation of the table will require relatively large joint loads due to the torque generated by the distance between the grasp and the center of mass of the table. Multiple manipulators however, may strategically place the grasps on the table so that the torque effects due to the difference between the center of mass 
of the table and the grasps are effectively eliminated. Systems with multiple manipulators also have the advantage of having larger works space areas by allowing the manipulators to pass objects to one another [2]. However the many advantages of using multiple manipulators for object manipulation, the path planning problem becomes increasingly more difficult due to the increased configuration space of the system being studied.

The goal of this research project is to develop a technique to generate a collision free path of an object from an initial state to a goal state using multiple robot manipulators without the need for a closed form inverse kinematic solution. The manipulators will maintain a fixed grasp on the object along the generated path such that there is assumed to be little to no relative transformation between the grasps on the object to be manipulated and the object itself. The manipulators and objects considered in the environments for this study are kinematic in nature. The dynamics and control laws for the manipulators to follow the generated paths are not considered in this thesis.

Traditionally, the forward kinematic and inverse kinematic solutions of robot manipulators are formulated using $4 \times 4$ transformation matrices [3]. This thesis uses dual quaternions to represent rigid body transformations as demonstrated by $[4,5]$. Dual quaternions offer the advantage over transformation matrices in that they are differentiable and thus provide a direct formulation to generating the Jacobian of a robotic manipulator. A numerical method can then be used where interpolations between transformation states is performed to generate an inverse kinematic solution using the Jacobian matrix of a robot manipulator at varying joint configurations.

Traditional Jacobian formulations transform the joint space of a manipulator to the three-dimensional translation space along with the three-dimensional orien- 
tation space of the end effector using Euler angles [3]. At certain configurations, Euler angles experience the phenomenon known as gimbal lock when the rotation axes align. Dual quaternion transformations do not suffer from this disadvantage.

Transformation rotation matrices describe the orientation of a body using a $3 \times 3$ matrix (composed of nine values). Unit quaternions represent the same orientation with only four values. Quaternions and dual quaternions take fewer operations to perform orientation and transformation concatenations as shown in $[6]$.

In this thesis, two algorithms are developed for the path planning of robot manipulators specifically for multiple manipulator systems attempting to transform a single object. This is performed without the need for a closed form inverse kinematic solution for a specific manipulator.

The first algorithm is a numerical inverse kinematic algorithm based upon the Jacobian of a robot manipulator. This algorithm takes as an input the desired end effector transformation as a dual-quaternion and returns a solution containing the joint angles of the manipulator. This solution method is unique in that a Jacobian formulation is developed from the joint space of the manipulator to the dual quaternion transform of the end effector rather than the traditional matrix transform. A damped-least square inverse method is then employed to avoid inverting a singular Jacobian matrix as well as damping the change in joint space to avoid diverging solutions. This allows for a general method for finding a satisfactory joint space solution that solves the desired end effector position and orientation that is applicable to manipulators of any joint space size, including redundant manipulators. The algorithm thus avoids the need to have a closed form solution for the manipulator being used in the system. Manipulators with revolute and/or prismatic joints are only considered in this thesis. 
The second algorithm is an adaptation of the sampling-based rapidly-exploring dense tree algorithm. The algorithm is adjusted to handle multiple manipulators transforming a single object while environment collisions and invalid joint spaces are avoided. The unique portion of this algorithm is that extensions for the tree are formed by extending the manipulators toward dual quaternion transformations of the object in the environment using the aforementioned numerical inverse kinematic algorithm. This avoids finding random joint configurations of each manipulator that satisfy the closed link constraints caused by the grasps of each manipulator on the object. The algorithm is able develop a path from an initial transformation to a goal transformation of an object where similar methods only find the desired end effector position while the orientation is not considered [7].

\subsection{Review of Literature}

Two highly researched methods for solving robotic path planning problems exist: rapidly-exploring dense trees (more specifically randomly-exploring random trees) and probabilistic road maps (or sampling-based road maps) [1]. Rapidlyexploring dense trees and probabilistic road map algorithms randomly sample the configuration space of the robot in its environment and attempts to connect these configurations from an initial configuration to a goal configuration while avoiding environment collisions and invalid joint spaces. Weghe et al [7], present an approach to the path planning problem for a single robot manipulator implementing a variation of a rapidly-exploring random tree algorithm with an optimal Jacobian transpose controller with a bias toward the goal configuration to achieve a faster solution by generating a certain percentage of configurations extending toward the goal configuration as presented in [1] and more efficient path planner than general rapidly-exploring random tree algorithms.

In the case of systems with multiple robot manipulators, a multi-robot rapidly- 
exploring random tree planner needs to be developed, where the controller handles multiple instances of the robot's inverse kinematic solvers and grasp qualities. Vahrenkamp et al [8] implemented an inverse reachability map for a mobile humanoid robot to enable the position of each robot to have a high probability of finding valid bi-manual grasps for a given object. A bi-manual, bi-directional rapidly-exploring random tree algorithm is used to find collision free paths of each manipulator to the object. This study focused on performing multiple grasps on an object, then manipulating the object via the mobile portions of the robots (e.g. grasping a large table with another robot, lifting it, then cooperatively moving to another room while avoiding obstacles).

Multiple grasps on a rigid body between two or more robot manipulators forms a closed linkage kinematic chain between the manipulators and the object. Yakey et al [9] address the planning problem for general linkages that form closed kinematic chains with redundant degrees of freedom by generating random samples in the closed chain collision free configuration space. A randomized error minimization technique is used to force as many of the random samples into the constrained configuration space as possible. Results of the planner with closed kinematic chains that had large amount of linkages performed well in two-dimensional space. However, solutions were found in the order of minutes to hours due to computationally intensive nature of generating valid random samples and connecting generated configurations.

Koga et al [2] present a method to manipulate an object with multiple manipulators. Robot tasks in this paper are split into two categories: transit paths and transfer paths. A transit path is defined as a manipulator path moving toward a goal configuration (e.g. to grab an object) where a transfer path is defined as manipulator(s) path(s) to move an object to a goal configuration. If the system 
encounters an invalid configuration with one or more robot, a re grasp of the object may be necessary and a new path will be required to be planned to move the object to the desired configuration.

Samavati et al [10] present a method for object grasp planning with multiple cooperative manipulators. A cooperation optimality grasp index is developed to judge cooperative grasp qualities. The three main criteria used to determine grasp quality in the algorithm presented are the static, kinetic and kinematic nature of the grasp and manipulator. Given the parameters of a sampled grasp set, a cost is associated with the grasp set. The purpose of the algorithm is to minimize the cost of the grasp sets. The best grasp sets are sought after by using a learning search routine to eliminate computationally costly grasp sets.

\subsection{Overview of Thesis}

In the following chapter, the methodology of the thesis is presented. At first, the method for which dual quaternions will represent rigid body transformations as well as the formulation of the dual quaternion Jacobian matrix is described. Following the discussion regarding transformations, a numerical inverse kinematic algorithm is presented which provides the joint angles given a desired end effector transformation. Finally a tree generation algorithm is presented which is adapted for the manipulation of a single object via multiple robot manipulators. This algorithm is designed to generate configurations of the manipulators that will transform an object from an initial configuration to a goal configuration where each configuration is connected to a previously established configuration in the tree.

The third chapter presents the findings of this thesis. Two separate simulations have been performed where the parameters of the presented algorithms have been varied to show their effect upon performance. Slight modifications to the environments such as removing obstacles along with changing the initial configu- 
rations of the manipulators have also been studied to determine the robustness of the algorithms. Sample paths have also been presented to show a visual representation of the manipulators transforming the object for each simulation from the initial transform to the goal transform.

The fourth chapter concludes the discussions presented in the previous chapters.

Appendix A includes a description of the forward kinematics as well as the Jacobian formulation of the Schunk LWA3 manipulator. Dimensions used for the forward kinematics as well as the geometries for collision detection were obtained from [11]. The geometries of the manipulator have been simplified to facilitate the creation of the collision model.

\section{List of References}

[1] S. M. LaValle, Planning Algorithms. Cambridge University Press, 2006.

[2] Y. Koga and J.-C. Latombe, "On multi-arm manipulation planning," in , 1994 IEEE International Conference on Robotics and Automation, 1994. Proceedings, 1994, pp. 945-952 vol.2.

[3] J. J. Craig, Introduction to Robotics: Mechanics and Control, 3rd ed. Prentice Hall, 2004.

[4] A. Perez and J. M. McCarthy, "Dual quaternion synthesis of constrained robotic systems," in ASME Journal of Mechanical Design, vol. 126, no. 3, 2003, pp. 425-435.

[5] H.-L. Pham, V. Perdereau, B. Adorno, and P. Fraisse, "Position and orientation control of robot manipulators using dual quaternion feedback," in 2010 IEEE/RSJ International Conference on Intelligent Robots and Systems (IROS), 2010, pp. 658-663.

[6] B. Kenwright, "A beginners guide to dual-quaternions: what they are, how they work, and how to use them for 3d character hierarchies," in WSCG 2012 Communication Proceedings, Conference June 2012, 2012, pp. 1-13.

[7] M. Vande Weghe, D. Ferguson, and S. Srinivasa, "Randomized path planning for redundant manipulators without inverse kinematics," in 2007 7th IEEERAS International Conference on Humanoid Robots, 2007, pp. 477-482. 
[8] N. Vahrenkamp, E. Kuhn, T. Asfour, and R. Dillmann, "Planning multi-robot grasping motions," in Humanoid Robots (Humanoids), 2010 10th IEEE-RAS International Conference on. IEEE, 2010, p. 593600.

[9] J. Yakey, S. LaValle, and L. Kavraki, "Randomized path planning for linkages with closed kinematic chains," in IEEE Transactions on Robotics and Automation, vol. 17, no. 6, 2001.

[10] F. C. Samavati, S. A. A. Moosavian, and A. Nahvi, "Optimal grasp planning for cooperative manipulators," in Electrical Engineering (ICEE), 2011 19th Iranian Conference on. IEEE, 2011, p. 16.

[11] "Schunk PowerCube LWA3 datasheet," 2007. [Online]. Available: wiki.cc. gatech.edu/humanoids/images/6/60/LWA3-Datasheet_2007-11.pdf 


\section{CHAPTER 2 METHODOLOGY}

\subsection{Introduction}

The research presented is this thesis is split into three interrelated problems. The first is establishing a rigid body transformation representation that includes the translation and orientation of the body in the environment. The second is to determine a method for determining the inverse kinematics given a goal transformation. The third is to develop a method based upon the construction of a tree using an adaptation of a rapidly-exploring dense tree algorithm to determine a collision free path for multiple manipulators to transform an object from an initial state to a goal state.

A simulation framework has been developed to test the validity and performance of the proposed algorithms. A mathematics foundation library was implemented to represent dual quaternion transformations along with the storing of valid configurations in the form of vertices. Edges have also been implemented in this library to represent valid connections between vertices in the environment.

A sample robot manipulator based upon the Schunk LWA3 seven degree of freedom was implemented along with a single object to be transformed and multiple environment obstacles. The bodies in the environment have been constructed using a graphics rendering environment. The forward and inverse kinematics of the manipulator have been developed as presented in this thesis. A collision library has been implemented to facilitate the detection of collisions between the rigid bodies present in the the environment. The planning algorithm presented will then finally build a tree that connects the initial and goal state of the object while being manipulated by the two sample manipulators. A path searching algorithm has also been applied to find an efficient path of the tree from the initial state to 
the goal state.

\subsection{Transformation Representation}

The unit dual quaternion is chosen to represent the rigid body transformations for this thesis. A dual quaternion is an extension of dual number theory [1] where a dual number is composed of a real component and a dual component. Like dual numbers, dual quaternions are a composition of a real quaternion representing the orientation of the body and the dual component quaternion representing the translation of the body $[2,3,4]$. As a prerequisite, the reader should be familiar with the quaternion algebra and calculus as presented in $[2,3]$, however a brief overview will be presented in this thesis.

A quaternion can be represented as a tuple of four scalars $q \in \mathbb{R}^{4}$ or alternatively a data pair consisting of a scalar component $w$ and a three-dimensional vector component $\hat{\boldsymbol{v}}[3]$.

$$
q=[w, x, y, z]=w+x \hat{\boldsymbol{i}}+y \hat{\boldsymbol{j}}+z \hat{\boldsymbol{k}}=w+\hat{\boldsymbol{v}}
$$

Quaternion to quaternion addition and subtraction is simply the addition/subtraction of the respective elements of the quaternion [2].

$$
\begin{aligned}
& q_{a}+q_{b}=w_{a}+w_{b}+\hat{\boldsymbol{v}}_{a}+\hat{\boldsymbol{v}}_{b} \\
& q_{a}-q_{b}=w_{a}-w_{b}+\hat{\boldsymbol{v}}_{a}-\hat{\boldsymbol{v}}_{b}
\end{aligned}
$$

Quaternion multiplication is performed by the following element operations $[2]:$

$$
q_{a} q_{b}=\left(w_{a} w_{b}-\hat{\boldsymbol{v}}_{a} \cdot \hat{\boldsymbol{v}}_{b}\right)+\left(w_{a} \hat{\boldsymbol{v}}_{b}+w_{b} \hat{\boldsymbol{v}}_{a}+\hat{\boldsymbol{v}}_{a} \times \hat{\boldsymbol{v}}_{b}\right)
$$

Where $(\cdot)$ is the vector dot product and $(x)$ is the vector cross product. Note that the scalar component of the quaternion product is $w_{a} w_{b}-\hat{\boldsymbol{v}}_{a} \cdot \hat{\boldsymbol{v}}_{b}$ and the vector component is $w_{a} \hat{\boldsymbol{v}}_{b}+w_{b} \hat{\boldsymbol{v}}_{a}+\hat{\boldsymbol{v}}_{a} \times \hat{\boldsymbol{v}}_{b}$. The conjugate of a quaternion is defined by 
the following [2]:

$$
q^{*}=w-\hat{\boldsymbol{v}}
$$

The norm (specifically the euclidean norm) of a quaternion is defined by the following [2]:

$$
\|q\|=\sqrt{w^{2}+x^{2}+y^{2}+z^{2}}
$$

Following the definition of the conjugate and norm of a quaternion, the inverse of a quaternion is found by dividing the conjugate of the quaternion by the square of the norm of the quaternion [2]:

$$
q^{-1}=q^{*} /\|q\|^{2}
$$

For unit quaternions where the norm is equal to one $(\|q\|=1)$, the inverse is simply just the conjugate of the quaternion.

Unit quaternions are commonly used to represent the orientation and/or rotation of a rigid body. Unit quaternions describing the orientation of a body $\left(q_{r}\right)$ may be formulated using an axis-angle representation where $\theta$ is the scalar rotational displacement about the unit axis $\hat{\boldsymbol{v}}[2,3]$.

$$
q_{r}=\cos \left(\frac{\theta}{2}\right)+\hat{\boldsymbol{v}} \sin \left(\frac{\theta}{2}\right)
$$

A dual quaternion $Q \in \mathbb{R}^{8}$ is then defined as the sum of a rotational unit quaternion $\left(q_{r}\right)$ and the product of the dual number component $(\epsilon)$ and the translational quaternion $\left(q_{t}\right)$.

$$
Q=q_{r}+\epsilon q_{t}
$$

Note the dual number component has the property such that $\epsilon^{n}=0$ where $n$ is any real positive integer greater than one [1]. An example of a dual component for a dual quaternion is when $\epsilon=\left[\begin{array}{ll}\mathbf{0} & \mathbf{1} \\ \mathbf{0} & \mathbf{0}\end{array}\right][1]$. Where $\mathbf{0}$ is the empty quaternion 
$\mathbf{0}=[0,0,0,0]$ and $\mathbf{1}$ is the identity quaternion $\mathbf{1}=[1,0,0,0]$. Following this example, a dual quaternion may be alternatively expressed in matrix form.

$$
Q=\left[\begin{array}{cc}
q_{r} & q_{t} \\
\mathbf{0} & q_{r}
\end{array}\right]
$$

To complete the definition of a dual quaternion, the translational component of the dual quaternion is defined $[2,3]$ :

$$
q_{t}=\frac{1}{2} t q_{r}
$$

Where $t$ is a "pure" translational quaternion $[2,3]$ where the scalar component is equal to zero and the vector component is equal to the translation vector containing the $x, y$, and $z$ displacements $(t=0+x \hat{\boldsymbol{i}}+y \hat{\boldsymbol{j}}+z \hat{\boldsymbol{k}})$

Dual quaternion addition and subtraction are simply the quaternion addition/subtraction of their like components $[2,3]$ :

$$
\begin{aligned}
& Q_{a}+Q_{b}=q_{r_{a}}+q_{r b}+\epsilon\left(q_{t_{a}}+q_{t b}\right) \\
& Q_{a}-Q_{b}=q_{r_{a}}-q_{r_{b}}+\epsilon\left(q_{t_{a}}-q_{t b}\right)
\end{aligned}
$$

The inverse of a dual quaternion must satisfy the property $Q Q^{-1}=\mathbf{1}+\epsilon \mathbf{0}$ where $\mathbf{1}$ is the identity quaternion and $\mathbf{0}$ is the empty quaternion. The dual quaternion that satisfies the above property is as follows:

$$
Q^{-1}=q_{r}^{-1}-\epsilon\left(q_{r}^{-1} q_{t} q_{r}^{-1}\right)
$$

One operation used frequently in this thesis is the calculation of the norm of a dual quaternion consisting of the difference of two different dual quaternions. This operation is simply the sum of the norms of the difference between the rotation quaternions and the difference between the translation quaternions.

$$
\left\|Q_{a}-Q_{b}\right\|=\left\|q_{r_{a}}-q_{r_{b}}\right\|+\left\|q_{t_{a}}-q_{t b}\right\|
$$


This definition is used often in this thesis as a distance metric to evaluate the length between two transformations or dual quaternions.

Dual quaternion multiplication is presented below, reemphasizing that $\epsilon^{2}=0$ $[2,3]$ :

$$
Q_{a} Q_{b}=q_{r_{a}} q_{r_{b}}+\epsilon\left(q_{r_{a}} q_{t_{b}}+q_{r_{b}} q_{t_{a}}\right)
$$

Care should be taken to ensure the dual quaternions are multiplied in the correct order since dual quaternion multiplication, like matrix multiplication, is not commutative.

Similar to transformation matrices, dual quaternion transformations may be compounded to determine successive linkage transformations for a robot manipulator given the transformations of the individual linkages [5]. For example, the end effector transformation of an $n$ joint manipulator may be found by the following operation:

$$
{ }_{n}^{0} Q={ }_{1}^{0} Q{ }_{2}^{1} Q \cdots{ }_{n}^{n-1} Q
$$

Where ${ }_{b}^{a} Q$ represents the transformation from joint $a$ to joint $b$. However, unlike transformation matrices, quaternions and dual quaternions are differentiable. The dual quaternion velocity is presented below:

$$
\dot{Q}=\dot{q}_{r}+\epsilon \dot{q}_{t}
$$

The velocity of a unit quaternion representing the orientation of a rigid body is found by differentiating Equation 8.

$$
\dot{q}_{r}=\frac{1}{2} \dot{\theta}\left(-\sin \left(\frac{\theta}{2}\right)+\hat{\boldsymbol{v}} \cos \left(\frac{\theta}{2}\right)\right)+\dot{\hat{\boldsymbol{v}}} \sin \left(\frac{\theta}{2}\right)
$$

Note that for joints and linkages where the axis of rotation does not change (single degree of freedom joints such as revolute and prismatic joints) $\dot{\hat{\boldsymbol{v}}}=\mathbf{0}$ where $\mathbf{0}$ is the zero vector. The equation above then reduces to the following:

$$
\dot{q}_{r}=\frac{1}{2} \dot{\theta}\left(-\sin \left(\frac{\theta}{2}\right)+\hat{\boldsymbol{v}} \cos \left(\frac{\theta}{2}\right)\right)
$$


The velocity of the translational quaternion is found by differentiating the definition shown by Equation 11.

$$
\dot{q}_{t}=\frac{1}{2}\left(\dot{t} q_{r}+t \dot{q}_{r}\right)
$$

For joints where there is no relative translations between them (e.g. revolute joints) the equation above reduces to the following:

$$
\dot{q}_{t}=\frac{1}{2}\left(t \dot{q}_{r}\right)
$$

For joints where there is no relative rotation between them (e.g. prismatic joints) Equation 21 reduces to the following:

$$
\dot{q}_{t}=\frac{1}{2}\left(\dot{t} q_{r}\right)
$$

For fixed axis single degree of freedom revolute joints, the dual quaternion velocity may be written as follows:

$$
\begin{aligned}
\dot{Q} & =\frac{1}{2} \dot{\theta}\left(-\sin \left(\frac{\theta}{2}\right)+\hat{\boldsymbol{v}} \cos \left(\frac{\theta}{2}\right)\right)+\epsilon \frac{1}{4} \dot{\theta} t\left(-\sin \left(\frac{\theta}{2}\right)+\hat{\boldsymbol{v}} \cos \left(\frac{\theta}{2}\right)\right) \\
& =\dot{\theta}\left(j_{r}+\epsilon j_{t}\right)=\dot{\theta} J
\end{aligned}
$$

Where $j_{r}$ is defined as the rotational Jacobian, $j_{t}$ is defined as the translation Jacobian, $\dot{\theta}$ is the scalar angular velocity, and $J$ is defined as the dual quaternion Jacobian. The definition given by the equation above may be expanded using Equation 17 to develop a relationship between the change in joint space of the manipulator to the change in transformation space of the manipulator's end effector. First Equation 17 is differentiated.

$$
{ }_{n}^{0} \dot{Q}={ }_{1}^{0} \dot{Q}_{2}^{1} Q \ldots{ }_{n}^{n-1} Q+{ }_{1}^{0} Q{ }_{2}^{1} \dot{Q} \ldots{ }_{n}^{n-1} Q+\ldots+{ }_{1}^{0} Q{ }_{2}^{1} Q \ldots{ }_{n}^{n-1} \dot{Q}
$$

Substituting Equation 24 into equation above gives:

$$
\begin{aligned}
{ }_{n}^{0} \dot{Q} & =\dot{\theta}_{1}\left({ }_{1}^{0} J_{2}^{1} Q \ldots{ }_{n}^{n-1} Q\right)+\dot{\theta}_{2}\left({ }_{1}^{0} Q_{2}^{1} J \ldots{ }_{n}^{n-1} Q\right)+\ldots+\dot{\theta}_{n}\left({ }_{1}^{0} Q_{2}^{1} Q \ldots{ }_{n}^{n-1} J\right) \\
& =\dot{\theta}_{1} J_{1}+\dot{\theta}_{2} J_{2}+\ldots+\dot{\theta}_{n} J_{n}
\end{aligned}
$$


Noting that

$$
J_{m}=\left(\prod_{k=1}^{m-1}{ }_{k}^{k-1} Q\right){ }_{m}^{m-1} J\left(\prod_{k=m+1}^{n}{ }_{k}^{k-1} Q\right)
$$

or in expanded form $J_{1}={ }_{1}^{0} J_{2}^{1} Q \ldots{ }_{n}^{n-1} Q, J_{2}={ }_{1}^{0} Q{ }_{2}^{1} J \ldots{ }_{n}^{n-1} Q, \ldots J_{n}=$ ${ }_{1}^{0} Q{ }_{2}^{1} Q \ldots{ }_{n}^{n-1} J$ etc. $J_{m}$ is the concatenations of the $m$ joint linkage Jacobian and the remaining linkage kinematic transformations. The above equation may also be written in matrix vector form as shown by [5].

$$
{ }_{n}^{0} \dot{Q}=\left[\begin{array}{llll}
J_{1} & J_{2} & \ldots & J_{n}
\end{array}\right]\left[\begin{array}{c}
\dot{\theta}_{1} \\
\dot{\theta}_{2} \\
\vdots \\
\dot{\theta}_{n}
\end{array}\right]=\boldsymbol{J} \dot{\boldsymbol{\theta}}
$$

Where $\boldsymbol{J}$ is defined as the Jacobian matrix where the columns are the $J_{n}$ dual quaternions and $\dot{\boldsymbol{\theta}}$ is a vector containing each joint displacement velocities.

The Jacobian matrix is an eight row matrix with $n$ columns $\boldsymbol{J} \in \mathbb{R}^{8 \times n}$, where $n$ is the size of the manipulator joint space. If the size of the joint space is eight, the inverse of the Jacobian matrix may be simply calculated by performing the linear inverse of a square matrix. If the joint space is less than eight, the left pseudo-inverse technique may be used to determine the inverse Jacobian matrix $[6]$.

$$
\boldsymbol{J}^{\dagger}=\left(\boldsymbol{J}^{T} \boldsymbol{J}\right)^{-1} \boldsymbol{J}^{T}
$$

If the joint space is greater than eight, the right pseudo-inverse technique may be used to determine the inverse Jacobian matrix [6].

$$
\boldsymbol{J}^{\dagger}=\boldsymbol{J}^{T}\left(\boldsymbol{J} \boldsymbol{J}^{T}\right)^{-1}
$$

When determining the inverse of the Jacobian matrix, care must be taken such that the matrix is not at or near singularity. In the case where the matrix is singular or near singular, the Jacobian matrix may be modified to prevent the inversion of 
a singular matrix using the damped least squares method [7].

$$
\boldsymbol{J}^{\dagger}=\left\{\begin{array}{cc}
\left(\lambda I_{n}+\boldsymbol{J}\right)^{-1} & n=8 \\
\left(\lambda I_{n}+\boldsymbol{J}^{T} \boldsymbol{J}\right)^{-1} \boldsymbol{J}^{T} & n<8 \\
\boldsymbol{J}^{T}\left(\lambda I_{8}+\boldsymbol{J} \boldsymbol{J}^{T}\right)^{-1} & n>8
\end{array}\right\}
$$

Where $\lambda$ is the damped least square factor. $\lambda$ should be large enough to prevent the solution from divergence however not too large to significantly slow down the calculation of the solution. The damped least square factor used in this thesis for the Schunk LWA3 manipulator is 0.085 . This provided the best results when calculating the inverse kinematics of the manipulator as discussed in the next section.

Given the inverse Jacobian matrix $\boldsymbol{J}^{\dagger}$, the joint displacements or joint velocities may be calculated given the transformation displacement or transformation velocity respectively.

$$
\dot{\boldsymbol{\theta}}=\boldsymbol{J}_{n}^{\dagger 0} \dot{Q}
$$

Due to the nonlinear relationship between the joint space of most robot manipulators and the transformation space of the manipulator's end effector, the Jacobian matrix and thus the inverse Jacobian matrix is only valid for either small changes in the manipulator's joint space and/or the end effector transformation space. Therefore, when calculating the change in joint space due to a large transformation space change, the change in transformation space must be incremented and the Jacobian matrix recalculated at the subsequent configurations. One method for interpolating the change in transformation space is the Screw Linear Interpolation (ScLERP) method as presented in [8].

$$
Q=\operatorname{ScLERP}\left(t, Q_{a}, Q_{b}\right)
$$

In Equation 33, $Q_{a}$ is the start transformation, $Q_{b}$ is the end transformation, and $t$ is an interpolation factor where $t \in[0,1]$. If $t=0, Q=Q_{a}$. Furthermore if 
$t=1, Q=Q_{b}$. Depending on the value of $t$, the ScLERP algorithm will return an interpolated transformation between the start and end transformation. The interpolation of the two transforms will yield a new transform that is by distance approximately $t\left\|Q_{b}-Q_{a}\right\|$ far away from $Q_{a}$ where $\left\|Q_{b}-Q_{a}\right\|$ is the distance between the two dual quaternion transformations. At subsequent iterations, where the distance $\left\|Q_{b}-Q_{a}\right\|$ changes, the length of the new iteration from $Q_{a}$ will change. To allow for near constant distance iterations, the inputs to Equation 33 are modified as follows:

$$
Q=\operatorname{ScLERP}\left(\frac{t}{\left\|Q_{b}-Q_{a}\right\|}, Q_{a}, Q_{b}\right)
$$

Where the interpolation of the two transforms will yield a new transform that is by distance approximately $t$ far away from $Q_{a}$.

\subsection{Inverse Kinematics}

The inverse kinematics of a robot manipulator is the determination of the joint displacements of the manipulator given the desired transformation of the manipulator's end effector. The philosophy for generating an inverse kinematic solution is the opposite of the generation of a forward kinematic solution, where given the manipulator's joint displacements, the transformation of the end effector is to be solved. The inverse kinematic method used for this thesis uses the dual quaternion formulation discussed above to determine the joint displacements that obtain the desired end effector transformation. Presented here is the numerical inverse kinematic method to determine the joint displacements of a robot manipulator devloped for this thesis.

Given the desired transformation of the end effector $Q_{g}$, the forward kinematics of the manipulator from the global reference frame to the end effector $Q_{s}$ is calculated at the current joint configuration $\boldsymbol{\theta}_{s}$ using Equation 17. The change in 
transformation space $\delta Q$ is then calculated.

$$
\delta Q=Q_{g}-Q_{s}
$$

The change in joint space $\delta \boldsymbol{\theta}$ is then calculated by integrating Equation 32 with respect to time. The Jacobian inverse matrix is calculated using the damped least square method as shown by Equation 31 to facilitate a solution to converge toward the goal transformation by damping the change in joint displacement space as well as preventing the inversion of a singular matrix. Note that the following equation is only valid for small changes in the joint space/transformation space, however the damping of the inverse Jacobian matrix will increment the joint space toward the desired end effector transform $Q_{g}$ even if the change in transformation space is large and help prevent a diverging solution.

$$
\delta \boldsymbol{\theta}=\boldsymbol{J}^{\dagger} \delta Q
$$

The calculated change in joint space is then added to the current joint space to obtain a new joint configuration.

$$
\boldsymbol{\theta}=\boldsymbol{\theta}_{s}+\delta \boldsymbol{\theta}
$$

The forward kinematics of the manipulator is reevaluated to determine the new transform of the end effector $Q_{s}$. If $Q_{s}$ is sufficiently close to $Q_{g}$ such that the norm of $Q_{g}-Q_{s}$ is less than some threshold $\sigma_{i k}$, the current joint space is a satisfactory solution given the end effector goal transform. Otherwise Equations 35, 36, and 37 are repeated until $Q_{s}$ is sufficiently close. If no solution is found such that the number of iterations $k$ exceeds some maximum number of iterations $k_{\max }$ or the calculated joint values are not within the manipulator's joint limits, the algorithm will terminate and return a failure.

The numerical inverse kinematic pseudo-code algorithm is presented in Figure 1. 


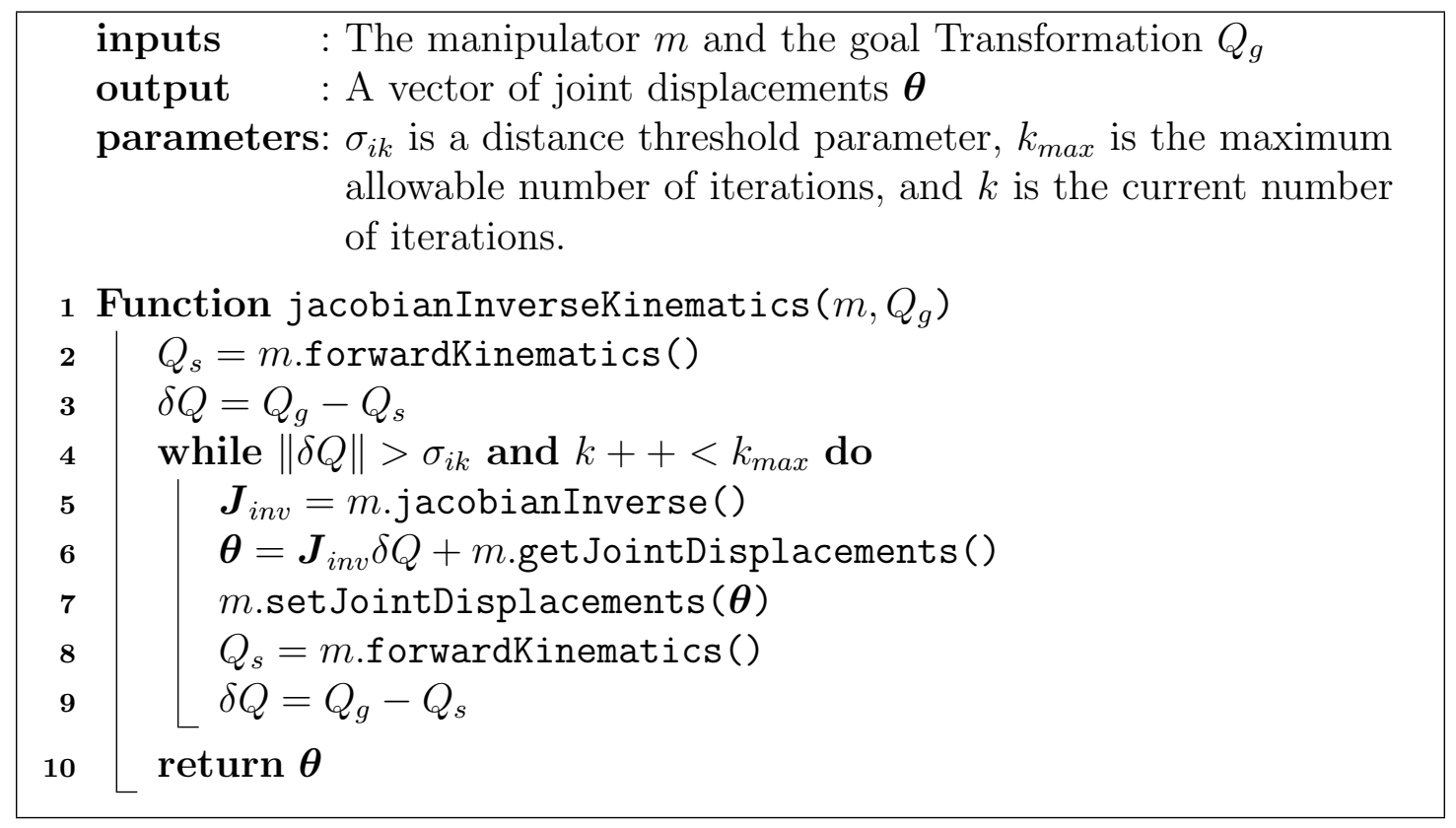

Figure 1: Numerical Inverse Kinematics Algorithm

\subsection{Cooperative Robot Manipulator Path Planning for the Transfor- mation of an Object}

For this thesis, a rapidly-exploring random tree [9] structured algorithm is developed to find a collision free path for the transformation of an object using multiple robot manipulators. The algorithm will take as inputs the object to be manipulated along with its desired goal transform and the grasps of the object by the manipulators and develop a tree that connects the input state with the goal state.

The tree is generated by adding vertices that contain the collision free joint displacements of the manipulators as well as the transform of the object in the environment. Edges are added to the tree to signify a connection between two vertices. The concept of vertices and edges may be visualized by referring to Figure 2 and 3 as provided by Figure 5.19 and 5.20 of [9].

The algorithm explores the environment space by randomly selecting object 


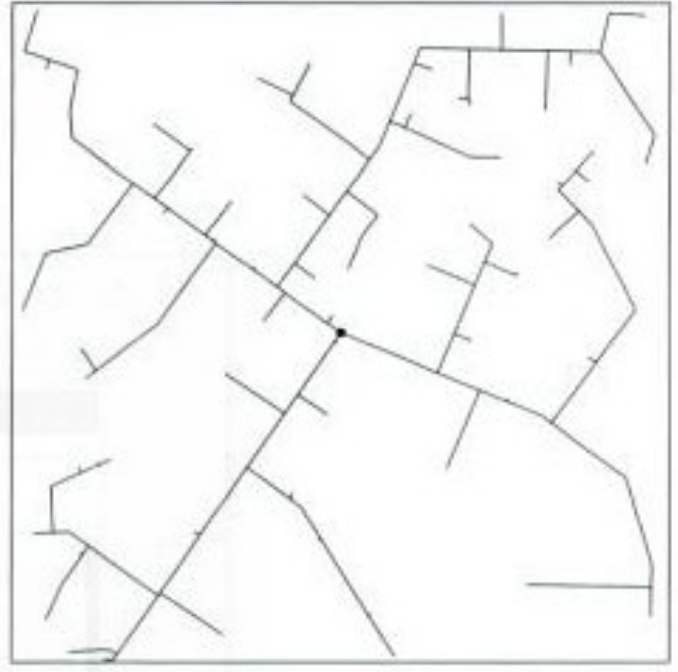

45 iterations

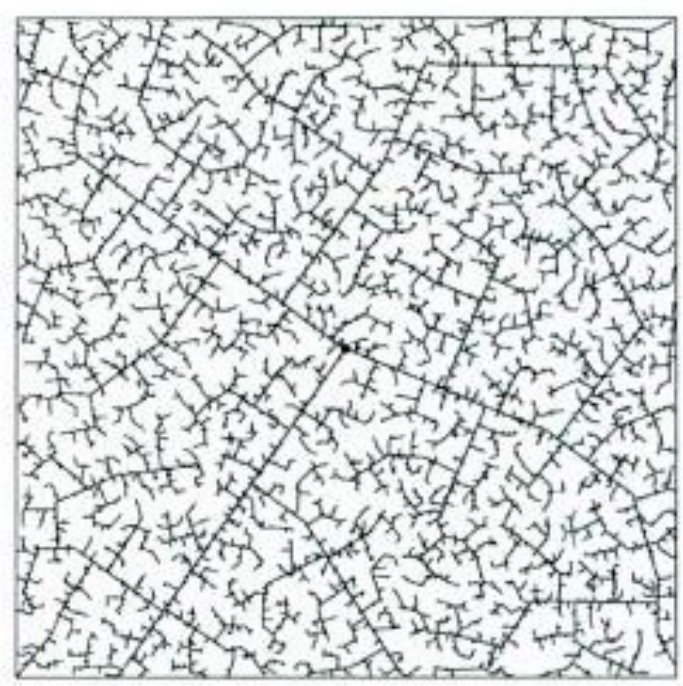

2345 iterations

Figure 2: Two Dimensional Configurations Space Rapidly-Exploring Dense Tree

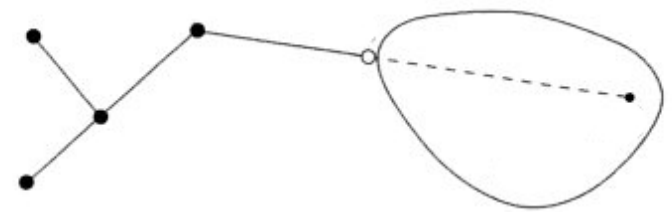

Figure 3: Visualization of Vertices and Edges for Tree Generation

transformations in the environment and displaces the joints of the manipulators to move the object toward the aforementioned transformation. The tree is formed so that the extensions to varying configurations are performed in small discrete "steps" such that the edges are kept small (the norm between the connected vertices is small). A small fraction of the iterations (along with the first iteration) will attempt to extend the tree toward the goal transform to introduce a bias toward finding a connection to the goal state [10].

The Jacobian inverse kinematic algorithm as presented in the previous section is used to determine the joint displacements given the transformation of the object. Due to the nonlinear nature of the Jacobian matrices, the extensions toward a new object transformation should be kept small so that the Jacobian can 
be recalculated. Furthermore, the edges of the tree are the transitions between the vertices of the tree. The edges in the tree should be kept small such that the distance between the two connected vertices is small. This will help minimize any relative motion between the grasps and the object along the path while the manipulators are between established vertices. The dual quaternion ScLERP algorithm as shown by Equation 34 is used to calculate the extended transformations.

After the generation of the tree composed of vertices and edges, a path searching algorithm is exercised to find an efficient path from the initial state to the goal state. Multiple "branches" may exist in the tree structure so the path searching may be non trivial. The algorithm implemented for this research is the $\mathrm{A}^{*}$ algorithm as presented in [11]. This technique assigns metrics to the vertices in the tree to determine an efficient path.

The algorithm implemented for this research has been developed upon the grounds of some assumptions. First, the obstacles in the environment are static. The only bodies that are considered dynamic for collision purposes are the manipulators and the object they are cooperatively manipulating. No relative motion between the grasps of the manipulators and the object is considered. Therefore, the grasps form a fixed rigid transformation between the end effector of the manipulator and the object. The workspaces of each manipulator must sufficiently overlap to the other manipulators via the grasps on the object such that a path is possible to obtain at the given configuration. Finally, this algorithm does not present a control system for the manipulators to follow the generated path.

Figure 4 presents the basic formulation of the rapidly-exploring dense tree for use in this thesis and is an extension of the rapidly-exploring dense tree algorithms of $[9]$.

The general outline for the rapidly-exploring dense tree is performed as follows. 
The tree $\tau$ is initialized with the intial configurations of the manipulators and object set to the current vertex $v_{s}$. The main loop of the algorithm is then entered and will break only if the goal configuration has been reached or the maximum number of iterations has been reached. The first action in the main loop is to calculate a random scalar from 0.0 to 1.0. If the random number is less than some goal bias or the main loop is in its first iteration, the extend configuration $v_{e}$ (the configuration the tree will attempt to extend toward) is set equal to the goal configuration $v_{g}$. Otherwise, the extend configuration $v_{e}$ is set to some random configuration in the environment. The nearest configuration present in the tree to the extend configuration is found and set to the current vertex $v_{s}$. The secondary extend loop is then entered and will exit the loop if the extend configuration has been reached. The first action performed in the secondary loop is to extend toward the extend configuration and set this configuration to the new configuration $v$. The extend function is designed so that small extensions are made toward the extend configuration $v_{e}$. If the new configuration $v$ does not cause a collision within the environment, the new vertex $v$ is added to the tree $\tau$ and an edge is added from the current configuration $v_{s}$ to the new configuration $v$. The current configuration $v_{s}$ is then set to the new configuration $v$. If the new configuration causes a collision in the environment, the secondary loop is terminated and reenters the main loop. Once the secondarly loop has been exited, the number of iterations will be incremented. If the goal configuration has been reached or the maximum number of iterations has been reached the main loop will terminate and finally return the generated tree $\tau$. 


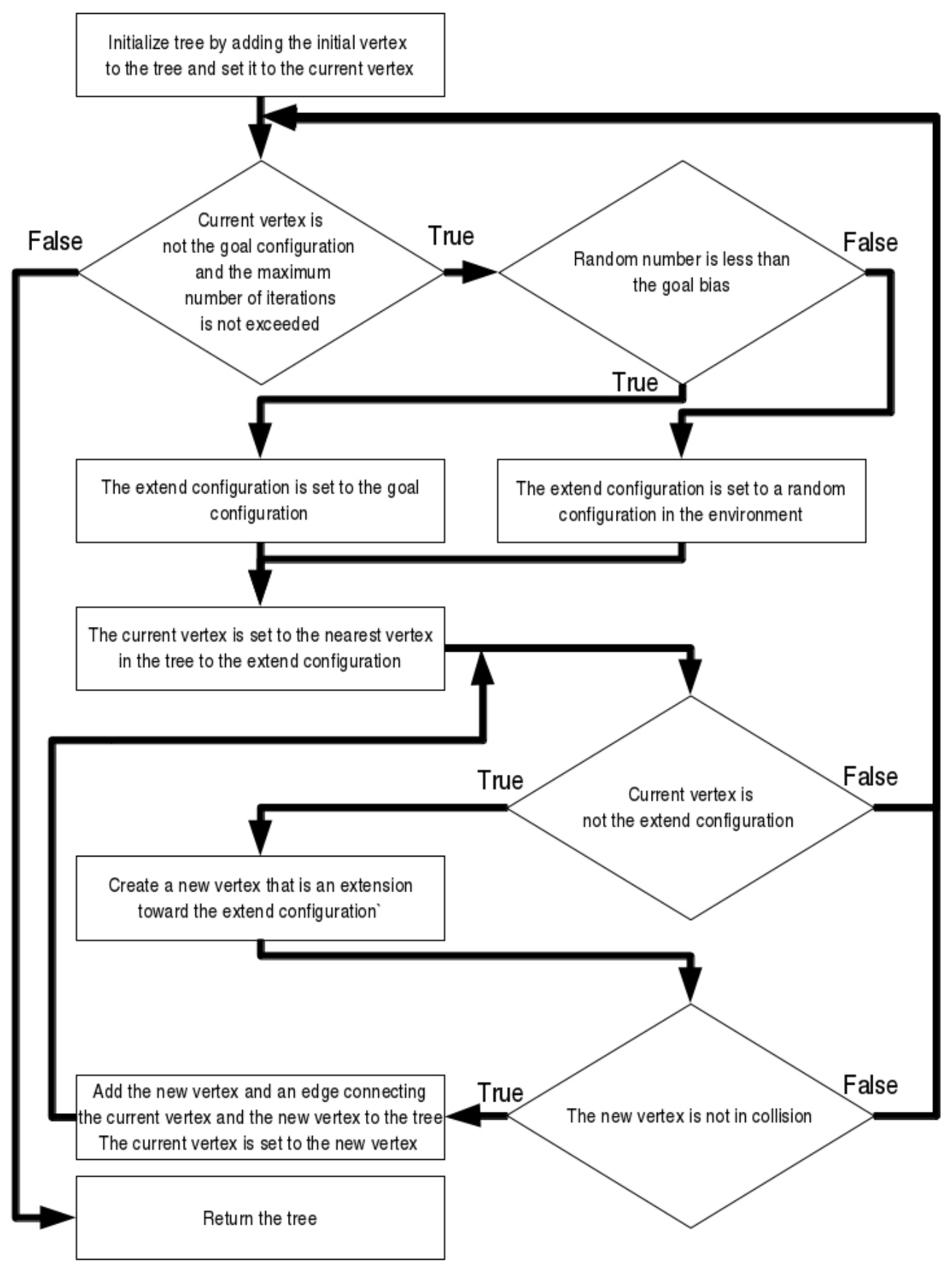

Figure 4: Rapidly-Exploring Dense Tree Flowchart

Continuing the discussion of the tree generation algorithm, the algorithm developed for generating a tree for this thesis has been implemented as shown by 
Figure 5. A few changes have been made to the algorithm in Figure 4 to handle multiple manipulators transforming a single object. Rather than finding random/goal configurations of each manipulator in the environment that satisfy the closed link constraints of the manipulators grasping a single object, random/goal transformations of the object in the environment are found. Equation 34 is used to determine an extend transform, which is a linear extension toward either a random transformation in the environment or the goal configuration. The algorithm presented by Figure 1 is then used given the extend transform of the object as an input to determine valid joint angles for each manipulator that is cooperatively transforming the object. The combination of the extended transform of the object along with the calculated joint angles for each manipulator are considered the extend configuration. Furthermore, rather than detecting if the current vertex is the extend/goal configuration, the algorithm only detects if the transform of the current vertex is close enough to the extend/goal transform. This allows for faster computation since the joint angles of the manipulators do not have to be compared at each configuration.

Similar to the outline as described in the algorithm above, the tree $\tau$ is first initialized by forming a vertex $v$ containing the initial configurations of the $m$ manipulators $\boldsymbol{\theta}_{1}, \boldsymbol{\theta}_{2}, \ldots, \boldsymbol{\theta}_{m}$ along with the initial transformation of the object $Q_{s}$. The vertex $v$ is then added to the tree $\tau$ and the current configuration $v_{s}$ is set to $v$. The next step in the initialization is to calculate the change in transformation space $\delta Q$ from the current object transform $Q_{s}$ to the goal transform $Q_{g}$. The norm of $\delta Q$ is calculated using Equation 15 and set equal to the distance metric $d_{0}$.

The main loop of the algorithm is then entered. The program will remain in the main loop as long as the distance metric $d_{0}$ is greater than the distance threshold parameter $\sigma_{\text {tree }}$ and the number of iterations $k$ does not exceed the 
maximum number of iterations $k_{\max }$. The first action in the main loop is to calculate a random scalar from 0.0 to 1.0 and compare the scalar to the goal bias. If the random scalar is less than the goal bias or the main loop has entered the first iteration such that $k=0$, the extend transformation $Q_{e}$ is set equal to the goal transformation $Q_{g}$. Otherwise, the extend transformation $Q_{e}$ is set equal to a random transformation in the environment. The tree configuration nearest the extend transform $Q_{e}$ is found and set to the current configuration $v_{s}$ with the current transform $Q_{s}$ set to the object transform of the $v_{s}$ configuration. The change in tranformation space $\delta Q$ from the current transform $Q_{s}$ to the extend transform $Q_{e}$ is then calulated. The norm of $\delta Q$ is calculated using Equation 15 and set equal to the distance metric $d_{1}$.

The secondary loop is then entered. The program will remain in the secondary loop as long as the distance metric $d_{1}$ is greater than the distance threshold $\sigma_{\text {tree }}$. The first action in the secondary loop is to set the object to the current object transform $Q_{s}$ and set the manipulators to the joint displacements stored in the current configuration $v_{s}$. The ScLERP algorithm as presented in Equation 34 is used to incrementally extend the object transform $Q$ to the extend transform $Q_{s}$. The object is then set to the extend transform $Q$. Each manipulator as present in the grasp list then calculates the respective manipulator's joint space $\boldsymbol{\theta}$ using the Jacobian inverse kinematics algorithm for the incremented object transform $Q$ as presented in the previous section. If the inverse kinematic calculation was successful for each manipulator, the calculated joint space $\boldsymbol{\theta}$ is set to its respective manipulator. However, if the inverse kinematic algorithm failed, another iteration of the main loop is restarted. The environment is then tested to see if any collisions are present. If no collision is present, a new vertex $v$ is created from the previously calculated manipulator joint spaces $\boldsymbol{\theta}_{1}, \boldsymbol{\theta}_{2}, \ldots, \boldsymbol{\theta}_{m}$ and the incremented transform 
$Q$. The vertex $v$ is added to the tree $\tau$ along with the edge from the current vertex $v_{s}$ to the newly added vertex $v$. The current vertex $v_{s}$ is then set to the new configuration $v$ and the current object transform $Q_{s}$ is set to the incremented object transform $Q_{s}$. The change in transformation space $\delta Q$ is then recalculated from the current transform $Q_{s}$ to the extend transform $Q_{e}$. The norm of the change in transformation space $\delta Q$ is reevaluated and set equal to the distance metric $d_{1}$. If the new configuration did cause a collision in the environment, the secondary loop is exited. After completion of the secondary loop, the change in transformation space $\delta Q$ from the current transform $Q_{s}$ to the goal transform $Q_{g}$ is reecalculated. The norm of the change in transformation space $\delta Q$ is reevaluated and set equal to the distance metric $d_{0}$. The number of main loop iterations $k$ is incremented.

Note that a grasp in the above algorithm is defined as a class that stores the object being manipulated, the manipulator, as well as the transform of the grasp relative to the transform of the object.

After an exit of the main loop, the tree $\tau$ is then returned by the algorithm. 


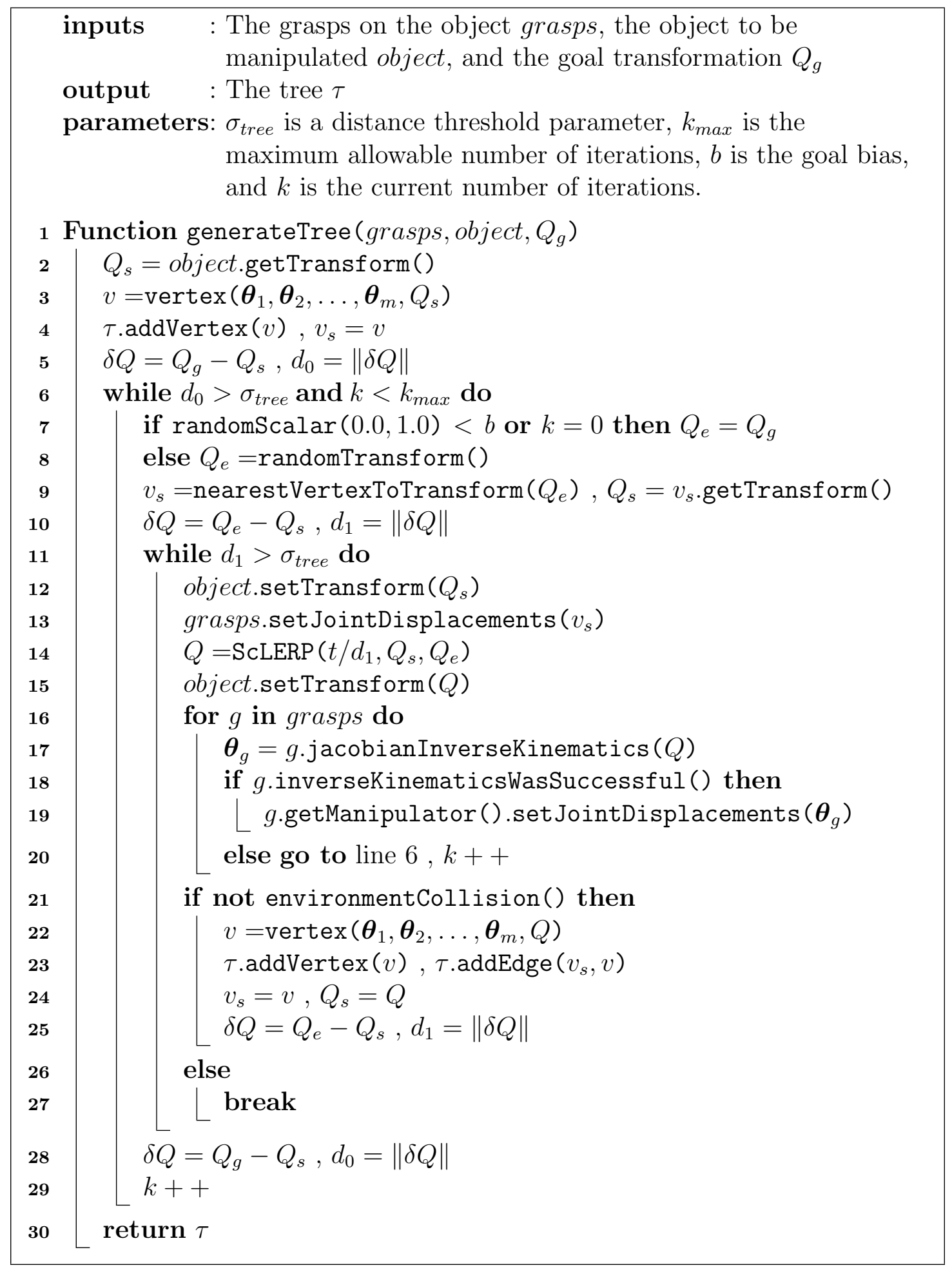

Figure 5: Tree Generation Algorithm 


\section{List of References}

[1] E. W. Weisstein. "Dual number - from wolfram MathWorld."

[2] D.-P. Han, Q. Wei, and Z.-X. Li, "Kinematic control of free rigid bodies using dual quaternions," in International Journal of Automation and Computing, vol. 5, no. 3, 2008, pp. 319-324.

[3] B. Kenwright, "A beginners guide to dual-quaternions: what they are, how they work, and how to use them for 3d character hierarchies," in WSCG 2012 Communication Proceedings, Conference June 2012, 2012, pp. 1-13.

[4] A. Perez and J. M. McCarthy, "Dual quaternion synthesis of constrained robotic systems," in ASME Journal of Mechanical Design, vol. 126, no. 3, 2003, pp. 425-435.

[5] H.-L. Pham, V. Perdereau, B. Adorno, and P. Fraisse, "Position and orientation control of robot manipulators using dual quaternion feedback," in 2010 IEEE/RSJ International Conference on Intelligent Robots and Systems (IROS), 2010, pp. 658-663.

[6] R. Penrose, "A generalized inverse for matrices," in Mathematical proceedings of the Cambridge philosophical society, vol. 51. Cambridge Univ Press, 1955, p. 406413.

[7] A. S. Deo and I. D. Walker, "Overview of damped least-squares methods for inverse kinematics of robot manipulators," in Journal of Intelligent and Robotic Systems, vol. 14, no. 1, 1995, pp. 43-68.

[8] X. Feng and W. Wan, "Dual quaternion blending algorithm and its application in character animation," in TELKOMNIKA Indonesian Journal of Electrical Engineering, vol. 11, no. 10.

[9] S. M. LaValle, Planning Algorithms. Cambridge University Press, 2006.

[10] M. Vande Weghe, D. Ferguson, and S. Srinivasa, "Randomized path planning for redundant manipulators without inverse kinematics," in 2007 7th IEEERAS International Conference on Humanoid Robots, 2007, pp. 477-482.

[11] W. Zeng and R. L. Church, "Finding shortest paths on real road networks: The case for a*," in International Journal of Geographical Information Science, vol. 23, no. 4, 2009, p. 531543. 


\section{CHAPTER 3}

\section{FINDINGS}

The algorithms presented in the previous section have been implemented in $\mathrm{C}++$ using an AMD Phenom II X6 3.2 GHz Processor on the Linux Mint 16 Operating System. Notable libraries used to facilitate the completion of this thesis include the Open Graphics Rendering Environment (OGRE) Library for visualizing the simulations, the Bullet Physics Engine Library for detecting environment collisions, as well as the Eigen Linear Algebra Library for linear system manipulation specifically for the implementation of matrix inversions. The global axes used in the environment align with the axes as shown in Figure 6.

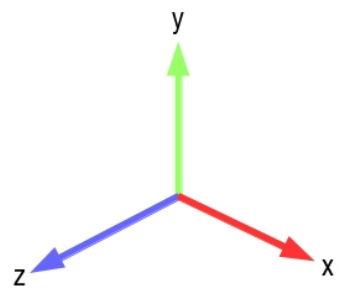

Figure 6: Graphics Rendering Global Axes

Two object transformation simulations were performed to examine the performance of the proposed algorithms with seven different parameter cases. Each case was run 100 times for each case of both simulations. The maximum number of iterations $k_{\max }$ was set to 10,000 for every simulation run. If the number of iterations exceeded that number, the execution of the tree generation was considered a failure. The parameters that will be varied for the different cases are the following: the maximum distance threshold for the tree generation $\sigma_{\text {tree }}$, the maximum distance threshold for the inverse kinematics $\sigma_{i k}$, the ScLERP interpolation factor $t$, as well as the goal bias $b$. The simulations were run an additional four 
times with the parameters from the first case however, the position of the second robot manipulator (the manipulator at the bottom right of the included figures in this chapter) was varied by 10 centimeters; along the $+x$ axis (case "a"), the $-z$ axis (case "b"), the $+x$ axis (case "c"), and finally the $+z$ axis (case "d"). Finally the first case of both simulation 1 and 2 was rerun with the obstacles in the environment removed.

Figure 7 presents the initial configuration as well as a sample goal configuration for the environments of each simulation. For both simulations, the robot manipulators are positioned 0.80 meters away from one another along the global $x$ axis. The objects and obstacles included in the two simulations are a small bench, a round table, and a square table. The small bench is 0.50 meters long, 0.20 meters wide and 0.32 meters high. The round table is 0.40 meters in diameter and is 0.22 meters high. The square table is 0.50 meters long and wide as well as 0.22 meters high. For collision detection purposes, all of the objects in the environment including the two robot manipulators are modeled as a series of rudimentary shapes. For example, the square table is a composition of a box and four cylinders. At the time of a collision detection query by the algorithm, the implemented collision detection will check for overlapping geometric pairs at their updated global transformations. In the event geometries overlap, a collision is present in the environment.

The first simulation is tasked with the transformation of the small bench from the center of the round table to square table. The transformation is simply the translation of the bench along the global negative $z$ axis.

The object of the second simulation is to flip the round table on the the square table upside down as well as translate the round table along the global $z$ axis. 


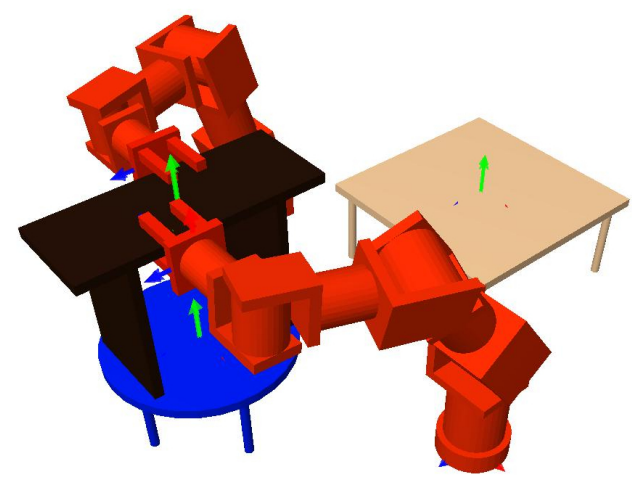

(a) Simulation 1 Initial Configuration

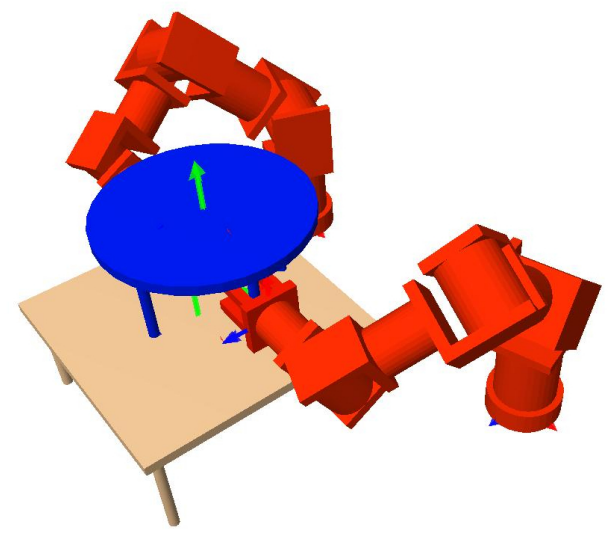

(c) Simulation 2 Initial Configuration

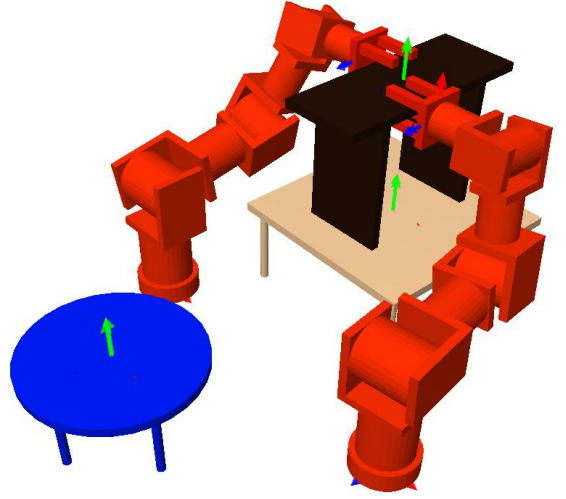

(b) Simulation 1 Goal Configuration

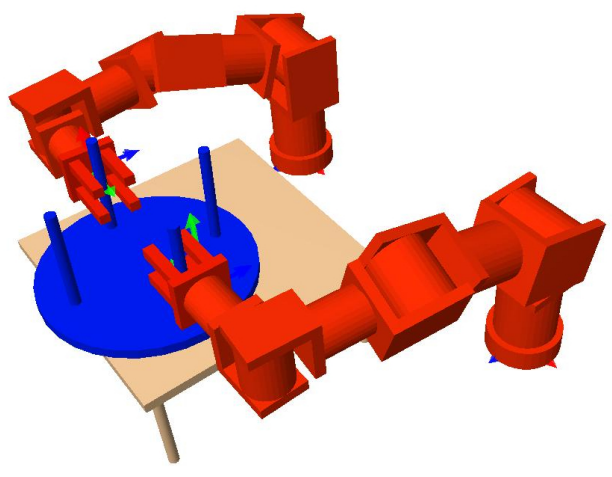

(d) Simulation 2 Goal Configuration

Figure 7: Simulations 1 and 2 Initial and Sample Goal Configurations

\subsection{Simulation 1 Results}

As discussed above, the objective of the first simulations is to translate the bench along the negative $z$ axis from atop the round table to the square table. After implementation of the presented algorithms for this environment a few observations regarding the performance of the algorithms have been observed.

Due to the location in the environment and kinematic properties of the robot manipulators, a simple translation of the bench was not achievable. This is due to the configuration space of the manipulators causing self-collisions and exceeding joint limits. The algorithm corrects for this by selecting random transforms for the 
bench where the manipulators will extend toward. Eventually the algorithm will have generated a connected tree from the initial configuration of the environment to the goal transform of the bench.

The recorded results for the experiments of simulation 1 are presented in Tables 1 and 2. The tables include the performance of the algorithm in terms of execution time, number of iterations for the execution of the tree, the number of collision free vertices generated by the tree, and finally the number of collision free vertices the manipulators will move along to transform the object from the initial state to the goal state.

\begin{tabular}{|l|c|c|c|c|c|c|c|c|c|}
\hline Case & 1 & 2 & 3 & 4 & 5 & 6 & 7 & 8 & 9 \\
\hline Threshold $\sigma_{\text {tree }}(\mathrm{m})$ & 0.01 & 0.05 & 0.005 & 0.01 & 0.01 & 0.01 & 0.01 & 0.01 & 0.01 \\
\hline Threshold $\sigma_{i k}(\mathrm{~m})$ & 0.001 & 0.001 & 0.001 & 0.005 & 0.0005 & 0.001 & 0.001 & 0.001 & 0.001 \\
\hline ScLERP Factor $t$ & 0.01 & 0.01 & 0.01 & 0.01 & 0.01 & 0.015 & 0.005 & 0.01 & 0.01 \\
\hline Goal Bias $b(\%)$ & 10 & 10 & 10 & 10 & 10 & 10 & 10 & 15 & 5 \\
\hline \hline Successes & 95 & 98 & 39 & 74 & 91 & 55 & 90 & 90 & 89 \\
\hline Failures & 5 & 2 & 61 & 26 & 9 & 45 & 10 & 10 & 11 \\
\hline Average Time (s) & 1.65 & 1.03 & 0.61 & 0.74 & 1.41 & 0.80 & 2.15 & 1.52 & 0.98 \\
\hline Min Time (s) & 0.13 & 0.12 & 0.13 & 0.10 & 0.13 & 0.10 & 0.17 & 0.13 & 0.14 \\
\hline Max Time (s) & 39.75 & 24.74 & 2.04 & 7.74 & 51.65 & 14.03 & 71.81 & 74.87 & 10.79 \\
\hline Average Iterations & 212.7 & 135.7 & 80.4 & 183.6 & 172.0 & 120.4 & 218.9 & 193.2 & 131.9 \\
\hline Min Iterations & 4 & 3 & 8 & 5 & 8 & 6 & 9 & 5 & 5 \\
\hline Max Iterations & 4515 & 2743 & 286 & 1532 & 6080 & 1949 & 6336 & 9115 & 1348 \\
\hline Average Tree Vertices & 110.5 & 93.4 & 89.9 & 101.9 & 98.1 & 62.5 & 204.1 & 96.3 & 112.5 \\
\hline Min Tree Vertices & 39 & 37 & 40 & 46 & 36 & 27 & 81 & 40 & 47 \\
\hline Max Tree Vertices & 845 & 701 & 204 & 353 & 638 & 319 & 1269 & 827 & 594 \\
\hline Average Path Vertices & 50.4 & 46.4 & 52.3 & 55.3 & 48.7 & 33.1 & 99.5 & 50.3 & 51.7 \\
\hline Min Path Vertices & 35 & 35 & 39 & 42 & 5 & 7 & 77 & 38 & 29 \\
\hline Max Path Vertices & 79 & 92 & 78 & 94 & 70 & 50 & 162 & 78 & 84 \\
\hline
\end{tabular}

Table 1: Simulation 1 Tree and Path Generation Results with Modified Parameters 


\begin{tabular}{|l|c|c|c|c|c|c|}
\hline Case & 1 & $\mathrm{a}$ & $\mathrm{b}$ & $\mathrm{c}$ & $\mathrm{d}$ & No Obstacles \\
\hline Threshold $\sigma_{\text {tree }}(\mathrm{m})$ & 0.01 & 0.01 & 0.01 & 0.01 & 0.01 & 0.01 \\
\hline Threshold $\sigma_{i k}(\mathrm{~m})$ & 0.001 & 0.001 & 0.001 & 0.001 & 0.001 & 0.001 \\
\hline ScLERP Factor $t$ & 0.01 & 0.01 & 0.01 & 0.01 & 0.01 & 0.01 \\
\hline Goal Bias $b(\%)$ & 10 & 10 & 10 & 10 & 10 & 10 \\
\hline \hline Successes & 95 & 90 & 84 & 90 & 94 & 100 \\
\hline Failures & 5 & 10 & 16 & 10 & 6 & 0 \\
\hline Average Time $(\mathrm{s})$ & 1.65 & 0.80 & 0.66 & 0.67 & 1.69 & 1.33 \\
\hline Min Time (s) & 0.13 & 0.13 & 0.13 & 0.13 & 0.16 & 0.18 \\
\hline Max Time (s) & 39.75 & 7.51 & 3.32 & 4.50 & 51.73 & 33.75 \\
\hline Average Iterations & 212.7 & 113.1 & 89.2 & 97.2 & 201.4 & 142.0 \\
\hline Min Iterations & 4 & 6 & 8 & 11 & 11 & 7 \\
\hline Max Iterations & 4515 & 1002 & 512 & 710 & 5420 & 3861 \\
\hline Average Tree Vertices & 110.5 & 93.8 & 91.0 & 87.47 & 108.9 & 144.5 \\
\hline Min Tree Vertices & 39 & 41 & 42 & 36 & 44 & 47 \\
\hline Max Tree Vertices & 845 & 354 & 238 & 238 & 921 & 669 \\
\hline Average Path Vertices & 50.4 & 51.4 & 49.3 & 50.47 & 50.9 & 50.2 \\
\hline Min Path Vertices & 35 & 39 & 36 & 36 & 36 & 38 \\
\hline Max Path Vertices & 79 & 80 & 72 & 72 & 81 & 97 \\
\hline
\end{tabular}

Table 2: Simulation 1 Tree and Path Generation Results with Modified Location of Manipulator 2 and Removed Obstacles

By examination, it can be seen that the increased distance threshold $\sigma_{\text {tree }}$ in case 2 yielded $3 \%$ more successes than case 1 as well as decreased the average time of executing the algorithm by approximately $38 \%$ and reduced the amount of average iterations by approximately $36 \%$. This increase in algorithm performance comes at the price that the allowable error in the final object transformation is five times larger. On the other hand, the performance of case 3 is severely reduced at $59 \%$ less successes compared to case 1 , however the allowable error for the object is $50 \%$ smaller.

The modification of the $\sigma_{i k}$ parameter for case 4 and 5 yielded fewer successes than case 1 . Case 4 yielded $22 \%$ fewer successes than case 1 where case 5 yielded only $4 \%$ fewer successes than case 1 . The significant decrease in performance of case 4 may be attributed to an increased number of collisions between the gripper of the manipulators and the object due to the relaxed end effector transformation 
threshold. The inverse kinematic threshold for case 5 was $50 \%$ more restrictive than case 1 , which caused more failures of the inverse kinematic algorithm, however the performance was nearly identical to case 1.

Reducing the ScLERP constant $t$ decreased the distance of established edges, thus increasing the number of vertices in the tree and ultimately the path as compared to case 1 . However, the increase in $t$ led to a significant decrease in the performance of the algorithm. If the ScLERP constant $t$ is not sufficiently small, the iterations toward the goal transformation may not be damped and may overshoot such that $t / d_{1}>1$. This may be remedied by asserting that $t / d_{1} \leq 1$. A large $t$ will also increase the distance between established vertices in the tree which may cause undesirable behavior of the manipulators and object between established configurations therefore, $t$ should be selected to prevent overshoot of transformations.

Modification of the goal bias had a small negative effect on the success rate of the algorithm for both case 8 and 9; however, the decrease in the goal bias in case 9 led to a faster execution time as well as reduction in iterations. This can be attributed to the increased tendency of case 9 to explore the environment, searching for valid configurations, rather than be too focused on extending toward the goal configuration.

Transformation of the base of robot manipulator 2 in cases "a", "b", "c" and "d" did not yield any significant changes of the performance of the algorithm as shown by Table 2. However, the success rates of all cases decreased compared to case 1 though execution time improved aside from case "d". It should be noted that significant transformations may cause the manipulators shared workspace to significantly decrease in size and may also cause invalid initial configurations as well as an unreachable goal transform. This may cause a significant decrease in 
performance.

The removal of the square and round table significantly increased the performance of the algorithm. No failures were recorded, where in case 1, 5 failures were recorded.

Two sample paths of simulation 1 case 1 are presented by Figure 8 and Figure 10, the former representing the path with the least amount of path configurations while the latter represents the path with the most path configurations. The separation between the figures in the path is four vertices except for the last two which may be smaller than four if the number of vertices in the path is not divisible by four. Figures 9 and 11 show the joint angles of the manipulators along the generated path.

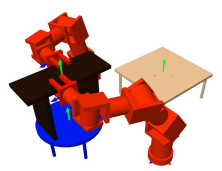

(a)

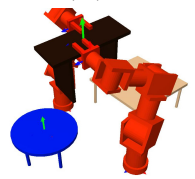

(d)

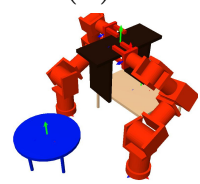

(g)

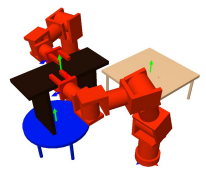

(b)

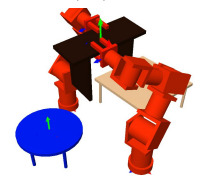

(e)

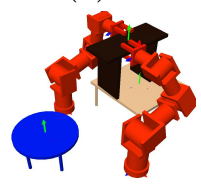

(h)

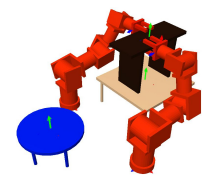

(j)

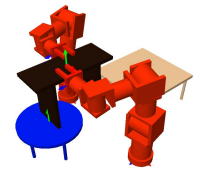

(c)

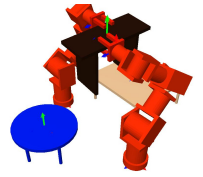

(f)

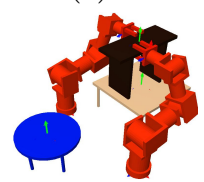

(i)

Figure 8: Simulation 1 Case 1 System Path with the Least Configurations 


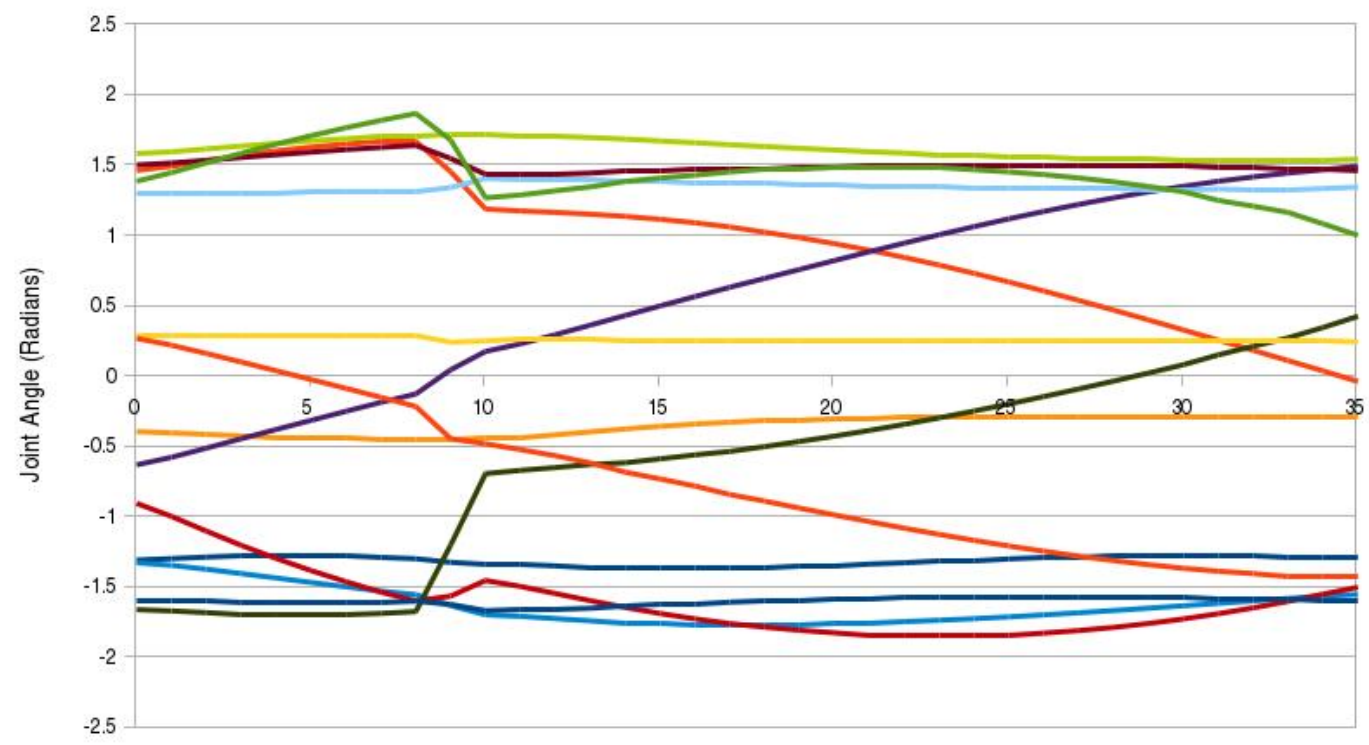

Configuration Number

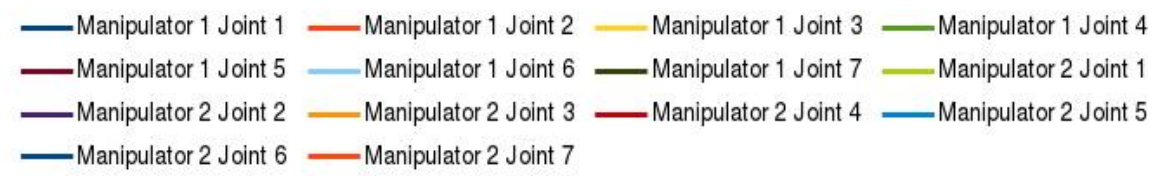

Figure 9: Simulation 1 Case 1 Joint Paths with the Least Path Configurations 


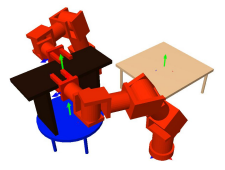

(a)

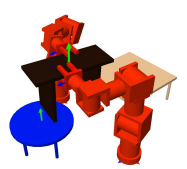

(d)

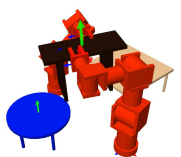

(g)

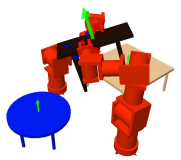

(j)

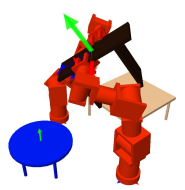

(m)

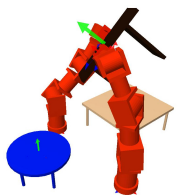

(p)

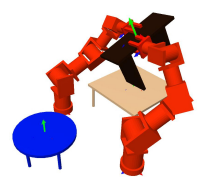

(s)

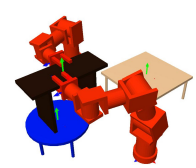

(b)

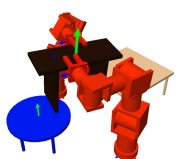

(e)

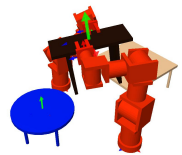

(h)

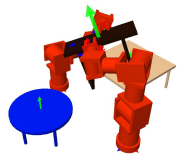

(k)

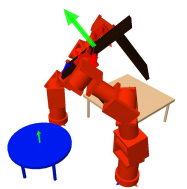

(n)

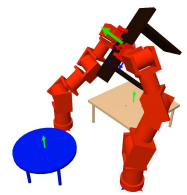

(q)

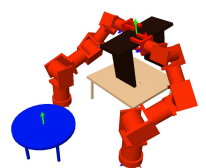

(t)

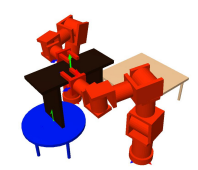

(c)

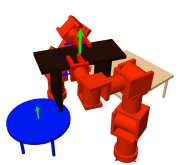

(f)

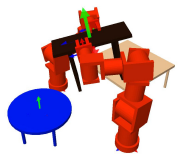

(i)

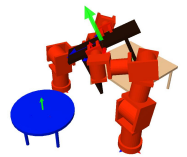

(1)

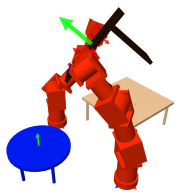

(o)

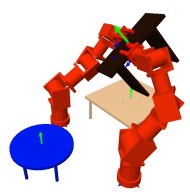

(r)

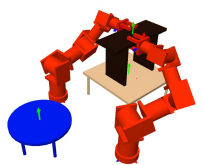

(u)

Figure 10: Simulation 1 System Path with the Most Configurations 


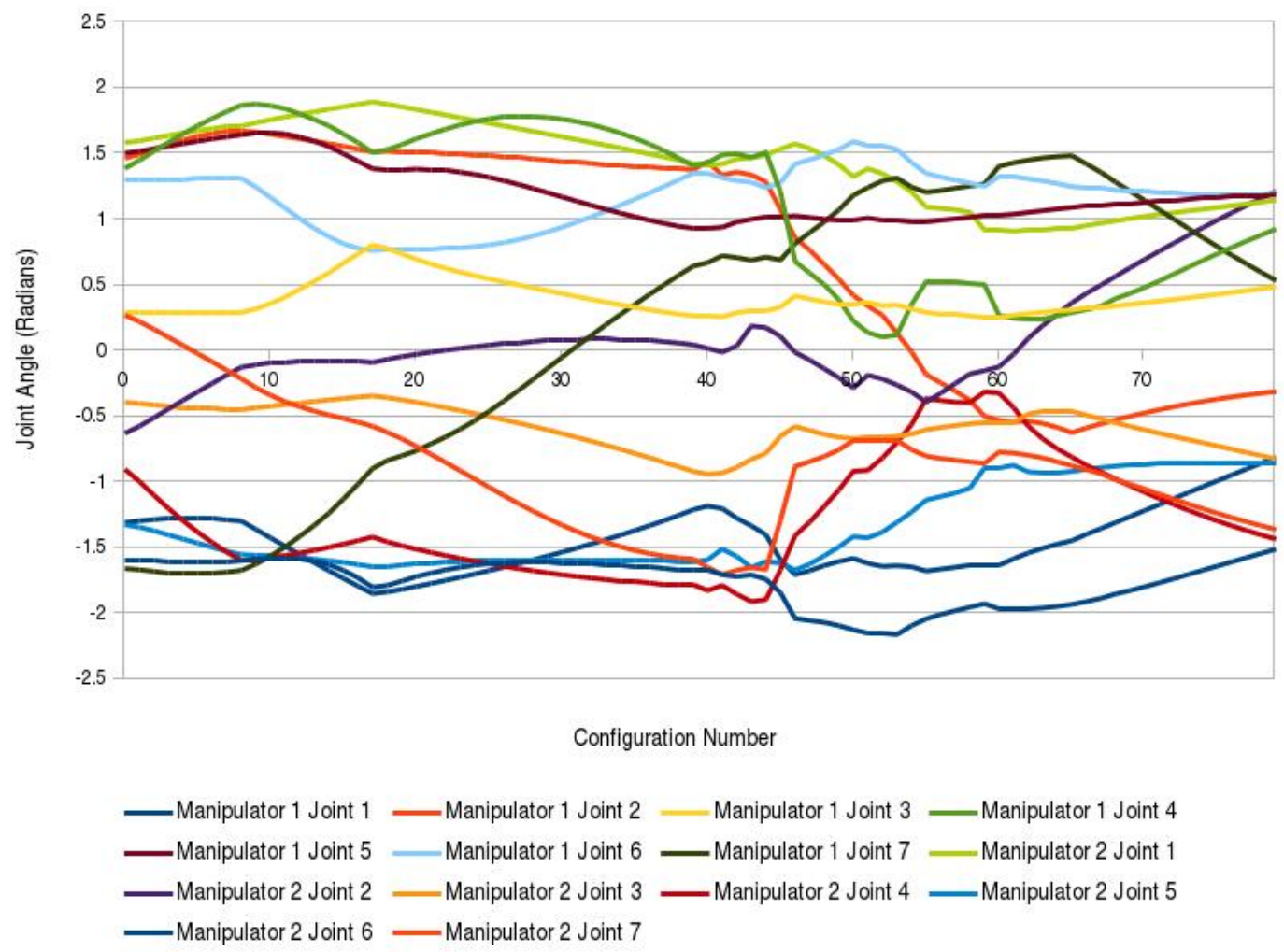

Figure 11: Simulation 1 Case 1 Joint Paths with the Most Path Configurations

\subsection{Simulation 2 Results}

The objective of the second simulations is to flip the round table upside down on top of the square table as well as translate it along the $z$ axis.

Due to the position of the square table underneath the round table, a direct transformation of the round table to the goal transformation is not possible without causing a collision between the square table and the round table. The algorithm corrects for this by searching for manipulator configurations that reorient the round table that ultimately leads the object to the goal transformation.

The recorded results for the experiments of simulation 2 are presented in Tables 3 and 4 . Similar to the previous section, the tables include the performance of the algorithm in terms of execution time, number of iterations for the execution 
of the tree, the number of collision free vertices generated by the tree, and finally the number of collision free vertices the manipulators will move along to transform the object from the initial state to the goal state.

\begin{tabular}{|l|c|c|c|c|c|c|c|c|c|}
\hline Case & 1 & 2 & 3 & 4 & 5 & 6 & 7 & 8 & 9 \\
\hline Threshold $\sigma_{\text {tree }}(\mathrm{m})$ & 0.01 & 0.05 & 0.005 & 0.01 & 0.01 & 0.01 & 0.01 & 0.01 & 0.01 \\
\hline Threshold $\sigma_{i k}(\mathrm{~m})$ & 0.001 & 0.001 & 0.001 & 0.005 & 0.0005 & 0.001 & 0.001 & 0.001 & 0.001 \\
\hline ScLERP Factor $t$ & 0.01 & 0.01 & 0.01 & 0.01 & 0.01 & 0.015 & 0.005 & 0.01 & 0.01 \\
\hline Goal Bias $b(\%)$ & 10 & 10 & 10 & 10 & 10 & 10 & 10 & 15 & 5 \\
\hline \hline Successes & 81 & 79 & 39 & 93 & 76 & 55 & 82 & 81 & 80 \\
\hline Failures & 19 & 21 & 61 & 7 & 24 & 45 & 18 & 19 & 20 \\
\hline Average Time (s) & 2.08 & 1.55 & 0.61 & 1.06 & 1.50 & 2.77 & 4.29 & 2.24 & 2.55 \\
\hline Min Time (s) & 0.21 & 0.17 & 0.13 & 0.13 & 0.27 & 0.14 & 0.25 & 0.20 & 0.19 \\
\hline Max Time (s) & 38.82 & 25.83 & 2.04 & 17.77 & 46.4 & 87.23 & 109.66 & 61.90 & 113.80 \\
\hline Average Iterations & 216.0 & 165.5 & 80.4 & 135.7 & 133.3 & 311.2 & 295.6 & 224.1 & 235.8 \\
\hline Min Iterations & 6 & 4 & 8 & 6 & 8 & 7 & 4 & 8 & 7 \\
\hline Max Iterations & 3986 & 2919 & 286 & 2135 & 4460 & 9103 & 6722 & 6109 & 9905 \\
\hline Average Tree Vertices & 129.2 & 112.2 & 89.9 & 125.1 & 114.9 & 92.3 & 287.7 & 117.7 & 125.8 \\
\hline Min Tree Vertices & 56 & 46 & 40 & 50 & 55 & 31 & 94 & 52 & 52 \\
\hline Max Tree Vertices & 691 & 573 & 204 & 692 & 686 & 781 & 2348 & 800 & 1173 \\
\hline Average Path Vertices & 57.8 & 53.6 & 52.3 & 59.3 & 56.1 & 39.0 & 113.7 & 57.5 & 56.45 \\
\hline Min Path Vertices & 47 & 23 & 39 & 48 & 47 & 19 & 94 & 47 & 27 \\
\hline Max Path Vertices & 78 & 149 & 78 & 173 & 76 & 75 & 161 & 156 & 106 \\
\hline
\end{tabular}

Table 3: Simulation 2 Tree and Path Generation Results with Modified Parameters 


\begin{tabular}{|l|c|c|c|c|c|c|}
\hline Case & 1 & $\mathrm{a}$ & $\mathrm{b}$ & $\mathrm{c}$ & $\mathrm{d}$ & No Obstacles \\
\hline Threshold $\sigma_{\text {tree }}(\mathrm{m})$ & 0.01 & 0.01 & 0.01 & 0.01 & 0.01 & 0.01 \\
\hline Threshold $\sigma_{i k}(\mathrm{~m})$ & 0.001 & 0.001 & 0.001 & 0.001 & 0.001 & 0.001 \\
\hline ScLERP Factor $t$ & 0.01 & 0.01 & 0.01 & 0.01 & 0.01 & 0.01 \\
\hline Goal Bias $b(\%)$ & 10 & 10 & 10 & 10 & 10 & 10 \\
\hline \hline Successes & 81 & 78 & 53 & 80 & 93 & 100 \\
\hline Failures & 19 & 22 & 47 & 20 & 7 & 0 \\
\hline Average Time $(\mathrm{s})$ & 2.08 & 3.82 & 3.64 & 2.16 & 2.39 & 0.15 \\
\hline Min Time (s) & 0.21 & 0.21 & 0.22 & 0.18 & 0.19 & 0.17 \\
\hline Max Time (s) & 38.82 & 113.99 & 73.24 & 55.77 & 54.16 & 1 \\
\hline Average Iterations & 216.0 & 360.9 & 344.5 & 215.2 & 247.8 & 1 \\
\hline Min Iterations & 6 & 9 & 11 & 8 & 7 & 1 \\
\hline Max Iterations & 3986 & 9934 & 6382 & 5554 & 5153 & 46 \\
\hline Average Tree Vertices & 129.2 & 158.2 & 143.0 & 123.25 & 130.1 & 46 \\
\hline Min Tree Vertices & 56 & 56 & 56 & 48 & 50 & 46 \\
\hline Max Tree Vertices & 691 & 1341 & 1155 & 961 & 933 & 46 \\
\hline Average Path Vertices & 57.8 & 57.7 & 56.9 & 58.0 & 59.7 & 46 \\
\hline Min Path Vertices & 47 & 46 & 13 & 45 & 48 & 46 \\
\hline Max Path Vertices & 78 & 121 & 103 & 147 & 133 & 46 \\
\hline
\end{tabular}

Table 4: Simulation 2 Tree and Path Generation Results with Modified Location of Manipulator 2 and Removed Obstacles

Similar to simulation 1 , the increased distance threshold $\sigma_{\text {tree }}$ in case 2 reduced the average execution time of the algorithms by $25 \%$; however, the success rate as well as the vertices in the tree and path did not significantly change. The reduced distance threshold significantly reduced the success rate as seen by the comparison of case 1 and case 3 .

The increase of the distance threshold $\sigma_{i k}$ for case 4 improved the success rate and execution time compared to case 1 . This can be attributed to the inverse kinematic algorithm being less restrictive, resulting in less inverse kinematic failures. This is the opposite effect that was shown by simulation 1 . The cause of this is due to the more forgiving grasp placements in simulation 2 . In simulation 1 , the grippers could only significantly translate along the $z$ axis and away from the bench without causing a collision between the gripper and the object. In simulation 2 , the grippers could significantly translate along the $y$ axis, move away from the 
table, along with rotate with respect to the axis created by the legs of the table without causing a collision between the gripper and the object. The reduction of $\sigma_{i k}$ had little effect on the performance of the algorithm.

As in simulation 1, the reduction of the ScLERP constant $t$ decreased the distance between the collision free vertices in the tree and path compared to case 1. The increase in the constant $t$ lead to performance losses compared to case 1 . This can be attributed to the overshoot of the goal transform as discussed in the previous section.

For this simulation, modification of the goal bias had little effect on the performance of the algorithm however the average execution time and iteration numbers of case 8 and 9 were slightly higher than case 1 .

Unlike simulation 1, the initial position of manipulator 2 had a significant impact on performance. For case "b" where the manipulator is translated by ten centimeters along the $-z$ axis, the number of successes decreased by $22 \%$. Since the manipulator was moved away from the object's initial and goal configuration, the valid configuration space for that manipulator was reduced. Unlike case "b", the case "d" scenario where the manipulator was moved closer to the object's initial and goal configuration improved the number of successes by $15 \%$ compared to case 1. Moving the manipulator along the $x$ axis did not significantly effect performance however the average execution time, iterations, and tree vertices increased in case "a" as compared to case 1.

The removal of the square table allowed the algorithm to extend vertices of the tree directly toward the object's goal transform without encountering any self collisions or invalid joint spaces which was the case for simulation 1. Therefore, the algorithm required only a single iteration to reach the goal transform in all attempts. 
Two sample paths of simulation 2 case 1 are presented by Figure 12 and Figure 14, the former representing the path with the least amount of path configurations while the latter represents the path with the most path configurations. The separation between the figures in the path is four vertices except for the last two which may be smaller than four if the number of vertices in the path is not divisible by four. Figures 13 and 15 show the joint angles of the manipulators along the generated path.

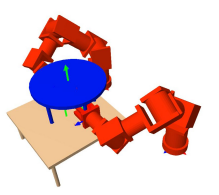

(a)

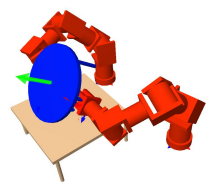

(d)

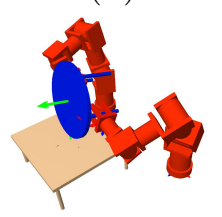

(g)

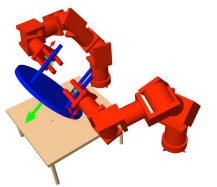

(j)

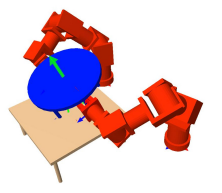

(b)

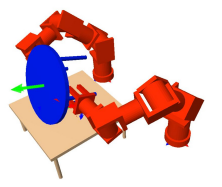

(e)

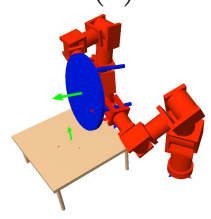

(h)

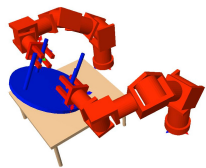

$(\mathrm{k})$

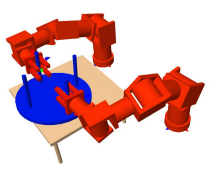

$(\mathrm{m})$

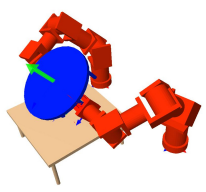

(c)

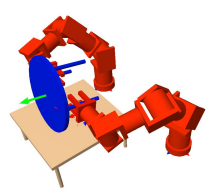

(f)

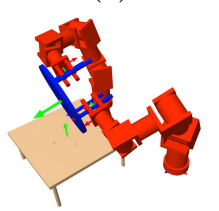

(i)

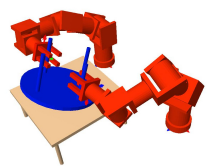

(l)

Figure 12: Simulation 2 System Path with the Least Configurations 


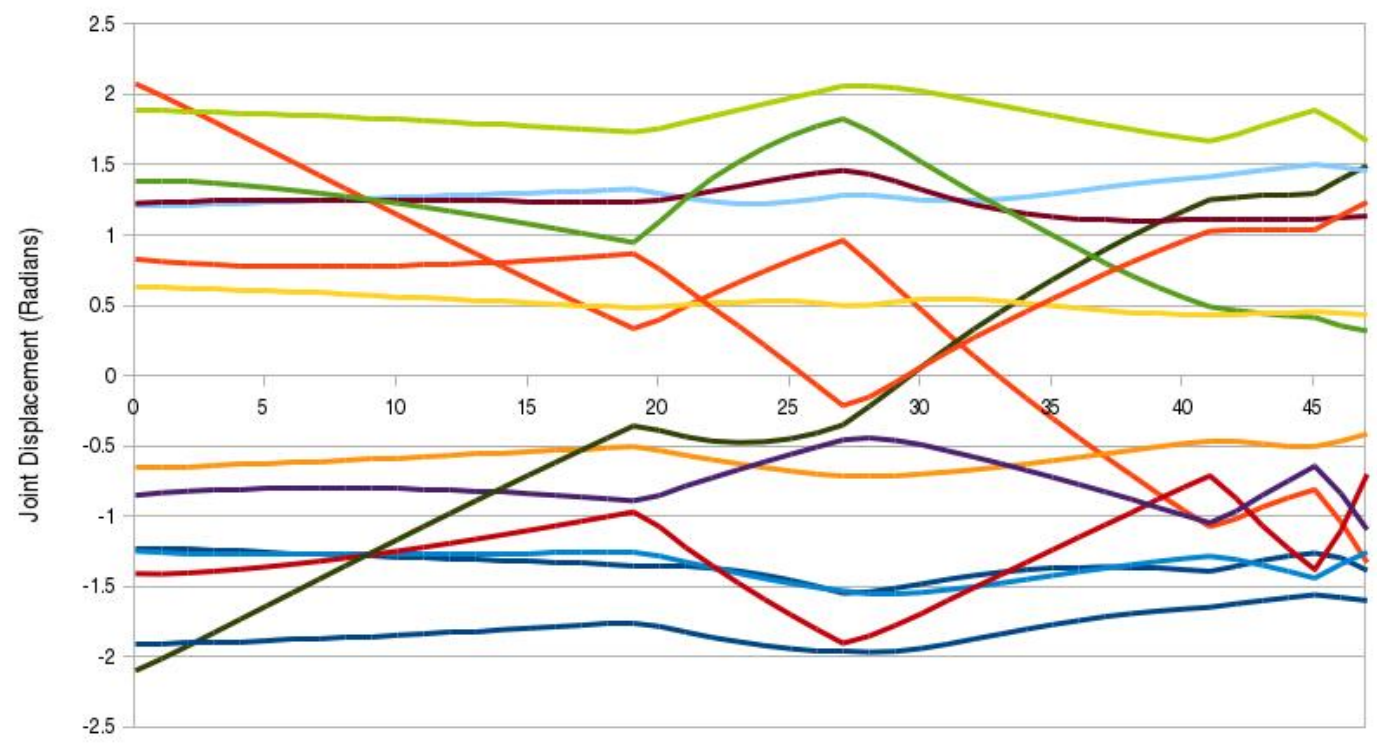

Configuration Number

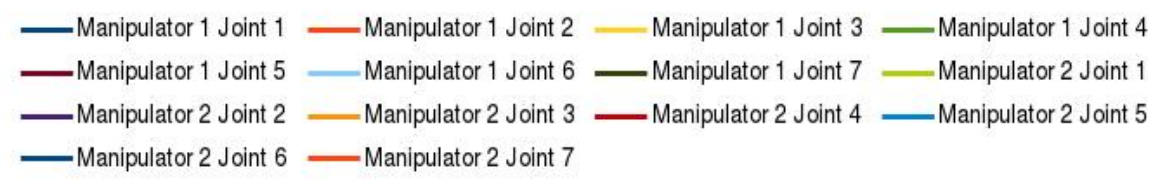

Figure 13: Simulation 2 Case 1 Joint Paths with the Least Path Configurations 


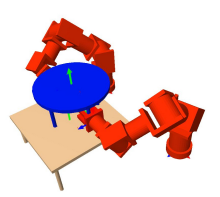

(a)

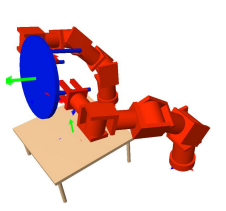

(g)

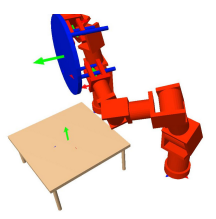

(m)

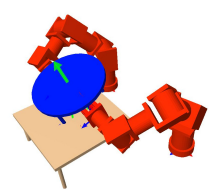

(b)

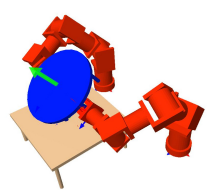

(c)

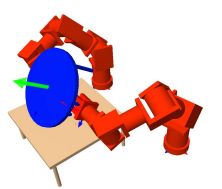

(d)

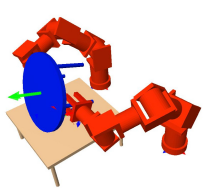

(e)

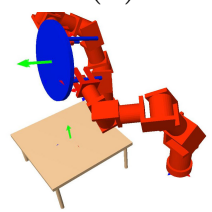

(j)

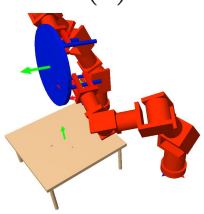

(k)

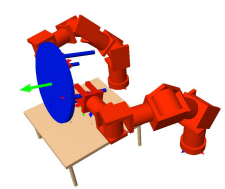

(f)

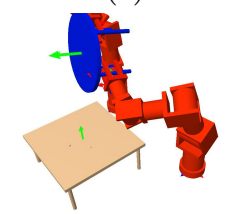

(1)

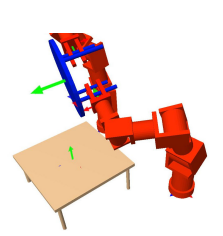

(n)

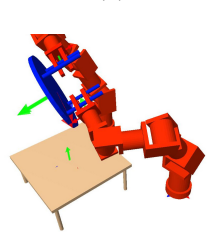

(o)

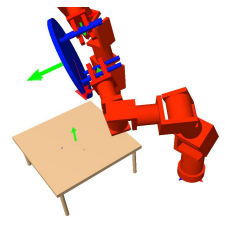

(p)

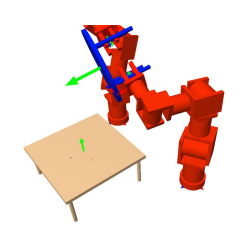

(q)

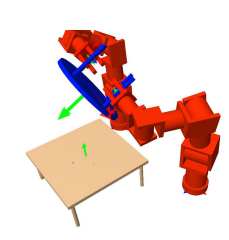

(r)

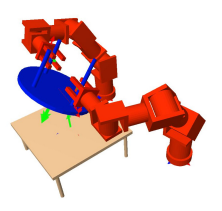

(s)

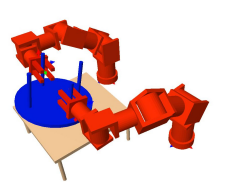

(t)

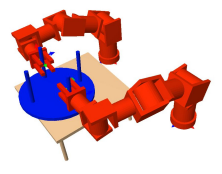

(u)

Figure 14: Simulation 2 System Path with the Most Configurations 


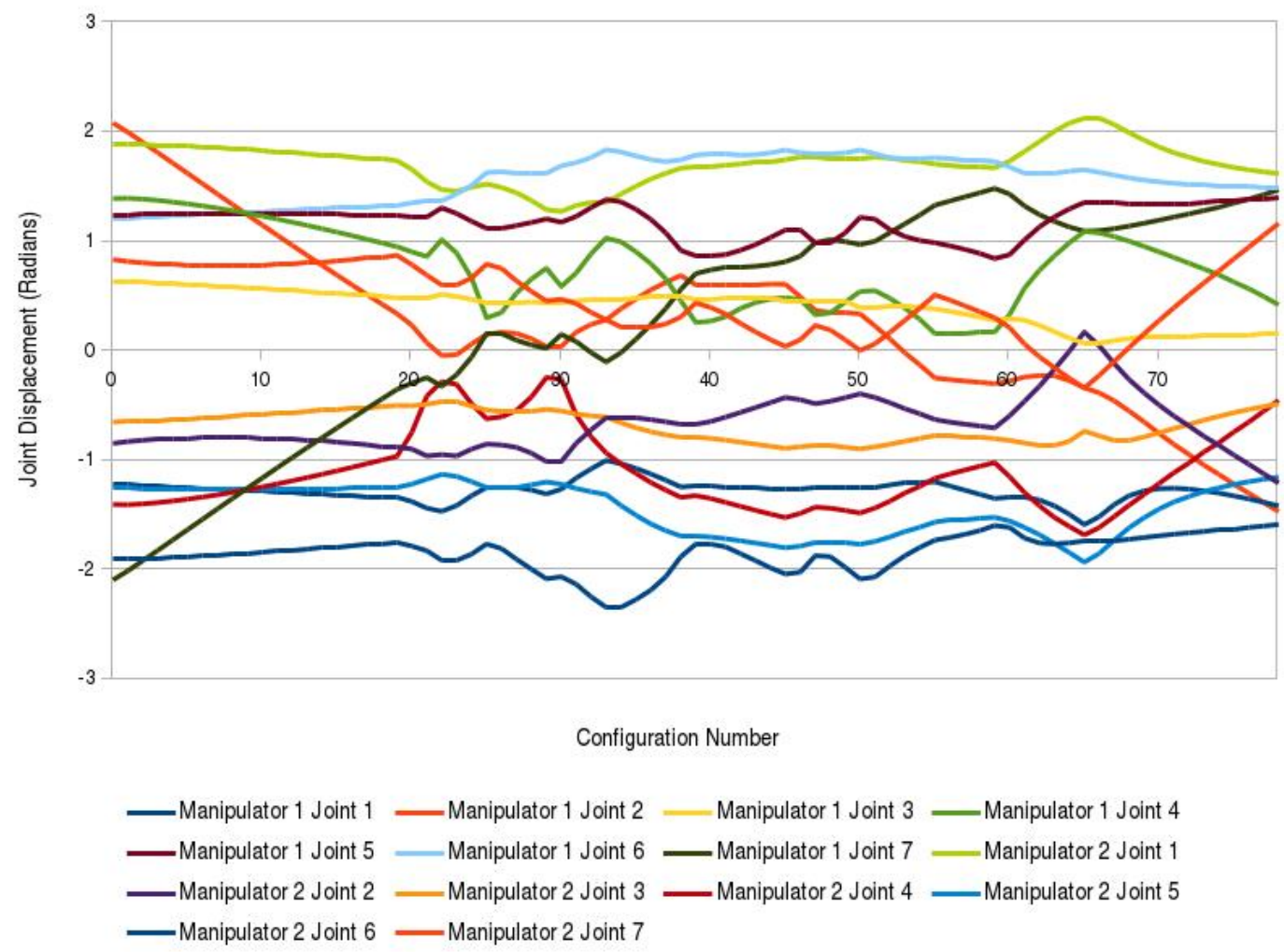

Figure 15: Simulation 2 Case 1 Joint Paths with the Most Path Configurations

\subsection{Remarks}

Figures $9,11,13$, and 15 show that the paths with least amount of configurations in the path have the smoothest joint paths. The joint paths of the larger path configurations have many unnecessary changes in direction. This is due to either the tree finding a path near the singular configurations of one or more of the manipulators or exploring an area in the environment where multiple collision bodies exist. This will prevent continuous extensions toward a transform in the environment and will cause the algorithm to extend toward a different transformation in the environment.

From both simulation experiments, a few observations can be made about the performance of the tree generation algorithm. First, the average execution time is 
roughly proportional to the average number of iterations such that approximately one second passes for every 100 iterations. Second, the number of vertices generated by the tree as well as present in the path is a function of the ScLERP factor t. Finally, the success rate of the algorithm may be improved by increasing the distance threshold $\sigma_{\text {tree }}$, however a larger error may occur between the goal vertex and the desired goal transformation. The distance threshold $\sigma_{i k}$ should be appropriately selected such that the inverse kinematic solution does not return failures due to over restrictive error allowances of the manipulator's end effector while not being too lenient such that a large error between the desired grasp transform and the actual grasp transform exist.

The results obtained in this thesis are compared to the rapidly-exploring random tree algorithm with an optimal Jacobian transpose controller (JT-RRT) as shown by Weghe et al [1]. Similar to the algorithm developed for this thesis, the JT-RRT algorithm is a sampling based algorithm, where the JT-RRT algorithm attempts to build a tree to find an adequate path for a single manipulator based upon the goal position of the end effector. The orientation of the end effector is not considered in this work. Out of six attempted simulation scenarios, the success rate of the JT-RRT algorithm was $99 \%$ with an average execution time of 1.90 seconds. The success rate of the algorithm presented by this thesis considering case 1 for simulations 1 and 2 was $88 \%$ with an average execution time of 1.87 seconds. The lower success rate of the presented algorithm can be attributed to the increased configuration space of the system, a smaller goal distance threshold (0.01 meters of case 1 compared to 0.15 meters), as well as accounting for the orientation of the end effector.

It can be observed from both sets of simulations, the variation of the algorithm parameters have significant effect on the performance of the algorithm. However, 
the parameter selection for one simulation environment may not be ideal for a different environment with different obstacles, number or type of manipulators etc. For the two simulations that were performed, the case 1 parameters provided reasonable success rates and execution time for both simulations considering the large configuration space composed of two seven degree of freedom robot manipulators. A few parameter selection guidelines should be followed for the best results. The distance threshold $\sigma_{\text {tree }}$ should be as large as possible without violating any tolerance requirements. This will give the algorithm the best chance for successfully determining a path to the goal transform. A distance threshold $\sigma_{i k}$ equal to 1 millimeter provided good results for the generation of the tree. The ScLERP factor $t$ should be kept low, however not so low that the execution time and number of vertices in the tree increases to an undesirable amount. $t$ equal to 0.01 provided good results for the tree generation. Best results for the success rate occurred with a goal bias of $10 \%$, therefore the goal bias should be selected at approximately $10 \%$.

\section{List of References}

[1] M. Vande Weghe, D. Ferguson, and S. Srinivasa, "Randomized path planning for redundant manipulators without inverse kinematics," in 2007 7th IEEERAS International Conference on Humanoid Robots, 2007, pp. 477-482. 


\section{CHAPTER 4}

\section{CONCLUSION}

In this thesis, an extension to the rapidly-exploring dense tree algorithm as well as a unique dual quaternion Jacobian based numerical inverse kinematic algorithm have been presented to find a valid path to transform an object from an initial configuration to a goal configuration using multiple robot manipulators. The tree generation algorithm is able to explore redundant manipulator configuration spaces while avoiding environment collisions and invalid manipulator configurations. Furthermore, the algorithm is performed without the need for a closed-form inverse kinematic solution of any specific type of robot manipulator.

The results obtained in the previous chapter show that, under well selected parameters, the tree generation algorithm performs well in obtaining a collision free path for multiple robot manipulators to transform an object from an initial state to a goal state in a kinematic environment. The system designer should experiment with the parameters of the algorithm as well as the grasps and location of the manipulators to obtain optimal performance. The tree generation algorithm may then be used as a planning tool in conjunction with appropriate sensors and controllers to complete a multiple manipulator object transformations system for use in real-time systems.

To further enhance the work of this thesis, a few extensions may be considered to add upon the current framework. A faster heuristic search method may be implemented to search for nearest vertices in the tree along with a more efficient/accurate distance metric. The algorithms presented may also be extended

to work in dynamic environments, as well as account for joint velocity limits of the present manipulators. A general joint torque controller to work cooperatively 
with the path generated will allow implementation in a real life setting for various manipulators. Combining a grasp planner with the tree generation algorithm will provide a more complete solution to cooperatively transforming an object along with the optimization of the generated paths specifically to remove any unnecessary motions generated by the presented algorithm. 


\section{APPENDIX}

\section{APPENDIX A}

\section{A.1 Forward Kinematics of the LWA3 Robot Manipulator}

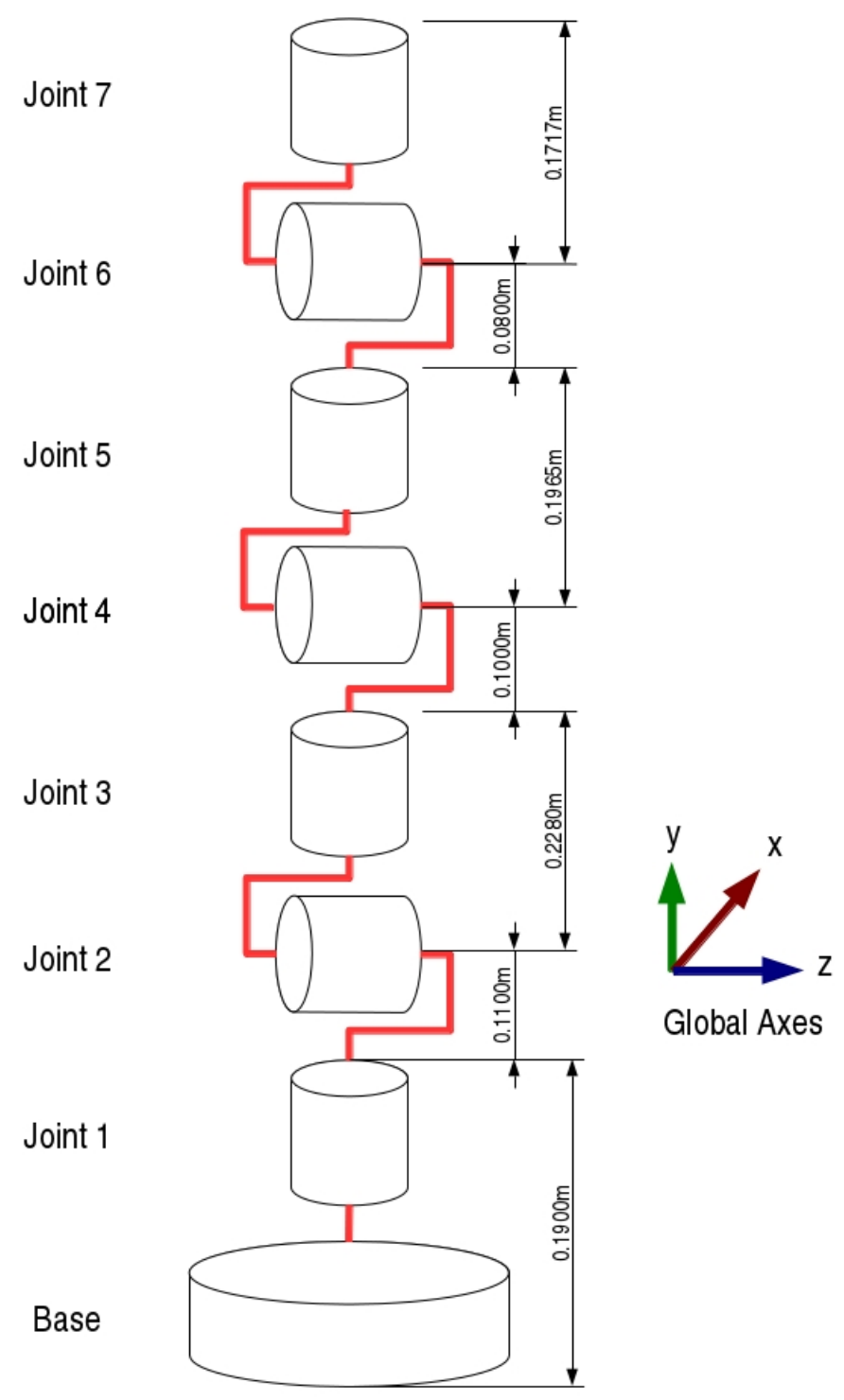

Figure A.1: Dimensions of the Schunk LWA3 Manipulator for Forward Kinematics 


\begin{tabular}{|c|c|}
\hline Joint Number & Joint Limit (Degrees) \\
\hline 1 & No Limit \\
\hline 2 & \pm 123.0 \\
\hline 3 & No Limit \\
\hline 4 & \pm 125.0 \\
\hline 5 & No Limit \\
\hline 6 & \pm 125.5 \\
\hline 7 & No Limit \\
\hline
\end{tabular}

Table A.1: Schunk LWA3 Joint Limits

The forward kinematics along with the joint limits of the Schunk LWA3 Manipulator have been implemented using the dimensions given by the Schunk data sheet [1] shown by Figure A.1 as well as Table A.1. The principal axes have been chosen so they align with the global axes in the graphics rendering environment. The transformations of each joint are derived using the dual quaternion formulation as shown by Equation 9 and are presented below.

$$
Q=q_{r}+\epsilon q_{t}=\left(\mathbf{1}+\epsilon \frac{1}{2} t\right) q_{r}
$$

Where $\mathbf{1}$ is the identity quaternion.

$$
\mathbf{1}=[1,0,0,0]
$$

The following definitions are for notational convenience:

$$
c_{n}=\cos \left(\theta_{n} / 2\right), s_{n}=\sin \left(\theta_{n} / 2\right)
$$

Where $\theta_{n}$ is the joint displacement of joint $n$.

The transformation from the base of the manipulator to joint 1 .

$$
{ }_{1}^{0} Q=\left(\mathbf{1}+\epsilon \frac{1}{2}[0,0,0.1900 m, 0]\right)\left[c_{1}, 0, s_{1}, 0\right]
$$


The transformation from joint 1 to joint 2 .

$$
{ }_{2}^{1} Q=\left(\mathbf{1}+\epsilon \frac{1}{2}[0,0,0.1100 m, 0]\right)\left[c_{2}, 0,0,-s_{2}\right]
$$

The transformation from joint 2 to joint 3 .

$$
{ }_{3}^{2} Q=\left(\mathbf{1}+\epsilon \frac{1}{2}[0,0,0.2280 m, 0]\right)\left[c_{3}, 0, s_{3}, 0\right]
$$

The transformation from joint 3 to joint 4 .

$$
{ }_{4}^{3} Q=\left(\mathbf{1}+\epsilon \frac{1}{2}[0,0,0.1000 m, 0]\right)\left[c_{4}, 0,0,-s_{4}\right]
$$

The transformation from joint 4 to joint 5 .

$$
{ }_{5}^{4} Q=\left(\mathbf{1}+\epsilon \frac{1}{2}[0,0,0.1965 m, 0]\right)\left[c_{5}, 0, s_{5}, 0\right]
$$

The transformation from joint 5 to joint 6 .

$$
{ }_{6}^{5} Q=\left(\mathbf{1}+\epsilon \frac{1}{2}[0,0,0.0800 m, 0]\right)\left[c_{6}, 0,0,-s_{6}\right]
$$

The transformation from joint 6 to joint 7 .

$$
{ }_{7}^{6} Q=\left(\mathbf{1}+\epsilon \frac{1}{2}[0,0,0.1717 m, 0]\right)\left[c_{7}, 0, s_{7}, 0\right]
$$

The transformation from the base of the manipulator to the end effector is found using Equation 17.

$$
{ }_{7}^{0} Q={ }_{1}^{0} Q_{2}^{1} Q_{3}^{2} Q_{4}^{3} Q_{5}^{4} Q_{6}^{5} Q_{7}^{6} Q
$$




\section{A.2 Jacobian Formulation of the LWA3 Robot Manipulator}

The Jacobian for each joint of the Schunk LWA3 Manipulator is found using Equation 24.

$$
\begin{aligned}
\dot{Q} & =\frac{1}{2} \dot{\theta}\left(-\sin \left(\frac{\theta}{2}\right)+\hat{\boldsymbol{v}} \cos \left(\frac{\theta}{2}\right)\right)+\epsilon \frac{1}{4} \dot{\theta} t\left(-\sin \left(\frac{\theta}{2}\right)+\hat{\boldsymbol{v}} \cos \left(\frac{\theta}{2}\right)\right) \\
& =\dot{\theta}\left(j_{r}+\epsilon j_{t}\right)=\dot{\theta} J
\end{aligned}
$$

The transformation velocity from the base of the manipulator to joint 1 .

$$
\begin{aligned}
{ }_{1}^{0} \dot{Q} & =\dot{\theta_{1}}\left(1+\epsilon \frac{1}{2}[0,0,0.1900 m, 0]\right)\left[-s_{1} / 2,0, c_{1} / 2,0\right] \\
& =\dot{\theta_{1}}\left({ }_{1}^{0} J\right)
\end{aligned}
$$

The transformation velocity from joint 1 to joint 2 .

$$
\begin{aligned}
{ }_{2}^{1} \dot{Q} & =\dot{\theta_{2}}\left(\mathbf{1}+\epsilon \frac{1}{2}[0,0,0.1100 m, 0]\right)\left[-s_{2} / 2,0,0,-c_{2} / 2\right] \\
& =\dot{\theta_{2}}\left({ }_{2}^{1} J\right)
\end{aligned}
$$

The transformation velocity from joint 2 to joint 3 .

$$
\begin{aligned}
{ }_{3}^{2} \dot{Q} & =\dot{\theta_{3}}\left(\mathbf{1}+\epsilon \frac{1}{2}[0,0,0.2280 m, 0]\right)\left[-s_{3} / 2,0, c_{3} / 2,0\right] \\
& =\dot{\theta}_{3}\left({ }_{3}^{2} J\right)
\end{aligned}
$$

The transformation velocity from joint 3 to joint 4 .

$$
\begin{aligned}
{ }_{4}^{3} \dot{Q} & =\dot{\theta}_{4}\left(\mathbf{1}+\epsilon \frac{1}{2}[0,0,0.1000 m, 0]\right)\left[-s_{4} / 2,0,0,-c_{4} / 2\right] \\
& =\dot{\theta}_{4}\left({ }_{4}^{3} J\right)
\end{aligned}
$$

The transformation velocity from joint 4 to joint 5 . 


$$
\begin{aligned}
{ }_{5}^{4} \dot{Q} & =\dot{\theta}_{5}\left(\mathbf{1}+\epsilon \frac{1}{2}[0,0,0.1965 m, 0]\right)\left[-s_{5} / 2,0, c_{5} / 2,0\right] \\
& =\dot{\theta}_{5}\left({ }_{5}^{4} J\right)
\end{aligned}
$$

The transformation velocity from joint 5 to joint 6 .

$$
\begin{aligned}
{ }_{6}^{5} \dot{Q} & =\dot{\theta_{6}}\left(1+\epsilon \frac{1}{2}[0,0,0.0800 m, 0]\right)\left[-s_{6} / 2,0,0,-c_{6} / 2\right] \\
& =\dot{\theta}_{6}\left({ }_{6}^{5} J\right)
\end{aligned}
$$

The transformation velocity from joint 6 to joint 7 .

$$
\begin{aligned}
{ }_{7}^{6} \dot{Q} & =\dot{\theta}_{7}\left(\mathbf{1}+\epsilon \frac{1}{2}[0,0,0.1717 m, 0]\right)\left[-s_{7} / 2,0, c_{7} / 2,0\right] \\
& =\dot{\theta}_{7}\left({ }_{7}^{6} J\right)
\end{aligned}
$$

The transformation velocity from the base of the manipulator to the end effector is found using Equation 25.

$$
\begin{aligned}
{ }_{7}^{0} \dot{Q} & =\dot{\theta}_{1}\left({ }_{1}^{0} J_{2}^{1} Q_{3}^{2} Q_{4}^{3} Q_{5}^{4} Q_{6}^{5} Q_{7}^{6} Q\right)+\dot{\theta}_{2}\left({ }_{1}^{0} Q_{2}^{1} J_{3}^{2} Q_{4}^{3} Q_{5}^{4} Q_{6}^{5} Q_{7}^{6} Q\right) \\
& +\dot{\theta}_{3}\left({ }_{1}^{0} Q_{2}^{1} Q_{3}^{2} J_{4}^{3} Q_{5}^{4} Q_{6}^{5} Q_{7}^{6} Q\right)+\dot{\theta}_{4}\left({ }_{1}^{0} Q_{2}^{1} Q_{3}^{2} Q_{4}^{3} J_{5}^{4} Q_{6}^{5} Q_{7}^{6} Q\right) \\
& +\dot{\theta}_{5}\left({ }_{1}^{0} Q_{2}^{1} Q_{3}^{2} Q_{4}^{3} Q_{5}^{4} J_{6}^{5} Q_{7}^{6} Q\right)+\dot{\theta}_{6}\left({ }_{1}^{0} Q_{2}^{1} Q_{3}^{2} Q_{4}^{3} Q_{5}^{4} Q_{6}^{5} J_{7}^{6} Q\right) \\
& +\dot{\theta}_{7}\left({ }_{1}^{0} Q_{2}^{1} Q_{3}^{2} Q_{4}^{3} Q_{5}^{4} Q_{6}^{5} Q_{7}^{6} J\right) \\
& =\dot{\theta}_{1} J_{1}+\dot{\theta}_{2} J_{2}+\dot{\theta}_{3} J_{3}+\dot{\theta}_{4} J_{4}+\dot{\theta}_{5} J_{5}+\dot{\theta}_{6} J_{6}+\dot{\theta}_{7} J_{7}
\end{aligned}
$$

The Jacobian matrix along with the joint velocity vector is then found using Equation 26. 


$$
{ }_{7}^{0} \dot{Q}=\left[\begin{array}{lllllll}
J_{1} & J_{2} & J_{3} & J_{4} & J_{5} & J_{6} & J_{7}
\end{array}\right]\left[\begin{array}{c}
\dot{\theta}_{1} \\
\dot{\theta}_{2} \\
\dot{\theta}_{3} \\
\dot{\theta}_{4} \\
\dot{\theta}_{5} \\
\dot{\theta}_{6} \\
\dot{\theta}_{7}
\end{array}\right]=\boldsymbol{J} \dot{\boldsymbol{\theta}}
$$

\section{List of References}

[1] "Schunk PowerCube LWA3 datasheet," 2007. [Online]. Available: wiki.cc. gatech.edu/humanoids/images/6/60/LWA3-Datasheet_2007-11.pdf 


\section{BIBLIOGRAPHY}

"Schunk PowerCube LWA3 datasheet," 2007. [Online]. Available: wiki.cc.gatech. edu/humanoids/images/6/60/LWA3-Datasheet_2007-11.pdf

Craig, J. J., Introduction to Robotics: Mechanics and Control, 3rd ed. Prentice Hall, 2004.

Deo, A. S. and Walker, I. D., "Overview of damped least-squares methods for inverse kinematics of robot manipulators," in Journal of Intelligent and Robotic Systems, vol. 14, no. 1, 1995, pp. 43-68.

Feng, X. and Wan, W., "Dual quaternion blending algorithm and its application in character animation," in TELKOMNIKA Indonesian Journal of Electrical Engineering, vol. 11, no. 10.

Han, D.-P., Wei, Q., and Li, Z.-X., "Kinematic control of free rigid bodies using dual quaternions," in International Journal of Automation and Computing, vol. 5, no. 3, 2008, pp. 319-324.

Kenwright, B., "A beginners guide to dual-quaternions: what they are, how they work, and how to use them for 3d character hierarchies," in WSCG 2012 Communication Proceedings, Conference June 2012, 2012, pp. 1-13.

Koga, Y. and Latombe, J.-C., "On multi-arm manipulation planning," in , 1994 IEEE International Conference on Robotics and Automation, 1994. Proceedings, 1994, pp. 945-952 vol.2.

LaValle, S. M., Planning Algorithms. Cambridge University Press, 2006.

Penrose, R., "A generalized inverse for matrices," in Mathematical proceedings of the Cambridge philosophical society, vol. 51. Cambridge Univ Press, 1955, p. 406413.

Perez, A. and McCarthy, J. M., "Dual quaternion synthesis of constrained robotic systems," in ASME Journal of Mechanical Design, vol. 126, no. 3, 2003, pp. 425-435.

Pham, H.-L., Perdereau, V., Adorno, B., and Fraisse, P., "Position and orientation control of robot manipulators using dual quaternion feedback," in 2010 IEEE/RSJ International Conference on Intelligent Robots and Systems (IROS), 2010, pp. 658-663.

Samavati, F. C., Moosavian, S. A. A., and Nahvi, A., "Optimal grasp planning for cooperative manipulators," in Electrical Engineering (ICEE), 2011 19th Iranian Conference on. IEEE, 2011, p. 16. 
Vahrenkamp, N., Kuhn, E., Asfour, T., and Dillmann, R., "Planning multi-robot grasping motions," in Humanoid Robots (Humanoids), 2010 10th IEEE-RAS International Conference on. IEEE, 2010, p. 593600.

Vande Weghe, M., Ferguson, D., and Srinivasa, S., "Randomized path planning for redundant manipulators without inverse kinematics," in 2007 7th IEEE-RAS International Conference on Humanoid Robots, 2007, pp. 477-482.

Weisstein, E. W. "Dual number - from wolfram MathWorld."

Yakey, J., LaValle, S., and Kavraki, L., "Randomized path planning for linkages with closed kinematic chains," in IEEE Transactions on Robotics and Automation, vol. 17, no. 6, 2001.

Zeng, W. and Church, R. L., "Finding shortest paths on real road networks: The case for a*," in International Journal of Geographical Information Science, vol. 23, no. 4, 2009, p. 531543 . 